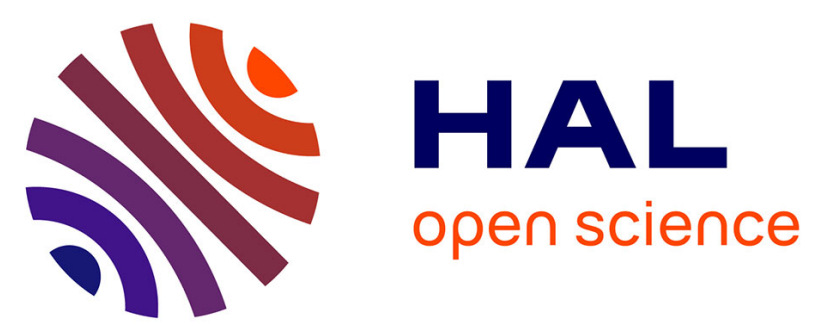

\title{
High prevalence of encoding RhoA-targeting toxin in clinical isolates of Staphylococcus aureus
}

Patrick Munro, René Clément, Jean-Philippe Lavigne, Céline Pulcini, Emmanuel Lemichez, Luce Landraud

\section{- To cite this version:}

Patrick Munro, René Clément, Jean-Philippe Lavigne, Céline Pulcini, Emmanuel Lemichez, et al.. High prevalence of encoding RhoA-targeting toxin in clinical isolates of Staphylococcus aureus: EDIN exotoxins in S. aureus infections. European Journal of Clinical Microbiology and Infectious Diseases, 2011, 30 (8), pp.965-972. 10.1007/s10096-011-1181-6 . hal-00669041

\section{HAL Id: hal-00669041 \\ https://hal.science/hal-00669041}

Submitted on 11 Feb 2012

HAL is a multi-disciplinary open access archive for the deposit and dissemination of scientific research documents, whether they are published or not. The documents may come from teaching and research institutions in France or abroad, or from public or private research centers.
L'archive ouverte pluridisciplinaire HAL, est destinée au dépôt et à la diffusion de documents scientifiques de niveau recherche, publiés ou non, émanant des établissements d'enseignement et de recherche français ou étrangers, des laboratoires publics ou privés. 
High prevalence of $e$ din- $C$ encoding RhoA-targeting toxin in clinical isolates of

\section{Staphylococcus aureus}

Patrick Munro ${ }^{1}$, René Clément ${ }^{1}$, Jean-Philippe Lavigne ${ }^{4,5}$, Céline Pulcini ${ }^{2,3}$, Emmanuel Lemichez $^{1^{*}}$ and Luce Landraud ${ }^{1,6^{*}}$

Running title: EDIN exotoxins in S. aureus infections

1 INSERM, U895, C3M, toxines microbiennes dans la relation hôte pathogènes, Université de Nice-Sophia-Antipolis, UFR Médecine, IFR50, Nice, F-06204, France

2 Université de Nice-Sophia-Antipolis, UFR Médecine, IFR50, Nice, F-06204, France

3 Service d'Infectiologie, Hôpital l'Archet 1, Route Saint Antoine de Ginestière, BP 3079 , 06202 Nice Cedex 3, France

4 INSERM, Espri 26, Université Montpellier 1, UFR de Médecine, Nîmes, France

5 Laboratoire de Bactériologie, CHU Caremeau, Nîmes, France

6 Laboratoire de Bactériologie, CHU de Nice, Hôpital l'Archet, Nice, France.

\section{* Corresponding authors:}

Luce Landraud, INSERM, U895, C3M, toxines microbiennes dans la relation hôte pathogènes, Université de Nice-Sophia-Antipolis, UFR Médecine, IFR50, Nice, F-06204, France, and Laboratoire de Bactériologie, CHU de Nice, Hôpital l'Archet, Nice, France.

Telephone: 0033489064261

Fax: 0033489064260

Mail: landraud.1@chu-nice.fr

Emmanuel Lemichez, INSERM, U895, C3M, toxines microbiennes dans la relation hôte pathogènes, Université de Nice-Sophia-Antipolis, UFR Médecine, IFR50, Nice, F-06204, France, and Laboratoire de Bactériologie, CHU de Nice, Hôpital l'Archet, Nice, France.

Telephone: 0033489064261

Fax: 0033489064260

\section{Mail: lemichez@unice.fr}

Abstract word count: 173 
1 Abstract Staphylococcus aureus, a major causative agent of human infection produces a large 2 array of virulence factor including various toxins. Among them, the host RhoA GTPase ADP3 ribosylating EDIN toxins are considered as potential virulence factors. Using the polymerase 4 chain reaction, we analyzed the virulence profile of $256 \mathrm{~S}$. aureus isolates from various 5 clinical sites of infections. We developed specific primers to detect the three isoforms of edin 6 encoding genes. We found a prevalence of 14\% (36 bacteria) of edin encoding genes among 7 these clinical isolates. Strikingly, we found that $90 \%$ of all edin-bearing S. aureus isolates 8 carried the type-C allele. Both the spa types and the profile of virulence factors of these edin9 positive isolates are highly variable. Notably, we show for the first time that edin-C positive isolates were more frequently recovered from deep-seated infections than other types of 11 infections. Our present work thus strongly suggests that presence of edin-C is a risk factor of S. aureus dissemination in tissues and thus represents a predictive marker for a pejorative 13 evolution of staphylococcal infections.

\section{Keywords}

18 Staphylococcus aureus, EDIN, toxin, ADP-ribosyltransferase, virulence factors, Rho 19 GTPases. 


\section{Introduction}

Staphylococcus aureus is a common bacterium, which is responsible for a unique variety of infections [1]. Development of pejorative forms of staphylococcal infections involves the combined action of numerous bacterial virulence factors, which corrupt host responses [2]. Bacterial virulence factors include specific adhesins, collectively referred as Microbial Surface Components Recognizing endothelial cell Adhesive Matrix Molecules (MSCRAMMs) and a large variety of toxins, such as the exfoliative toxins (ETs), hemolysin, leukocidin, enterotoxins and EDINs (epidermal cell differentiation inhibitors) [3-6].

EDINs belong to the family of Clostridium botulinum C3 exoenzyme [6, 7]. They are members of a group of major bacterial virulence factors targeting host Rho GTPases [4, 6-9]. Rho proteins control essential cellular processes such as cell polarity, movement and phagocytosis, as well as cohesion of intercellular junctions [6, 10, 11]. Recent findings suggest that EDINs might favor bacterial dissemination in tissues, by a haematogenous route, through induction of large transcellular tunnels in endothelial cells named macroapertures [12-14]. Indeed, recent data show that $S$. aureus EDIN toxin promotes formation of infection foci in a mouse model of bacteremia [15]. To date, three isoforms of EDIN have been characterized. These comprise the first discovered EDIN isoform (EDIN-A), isolated from the E-1 strain of S. aureus [16], as well as EDIN-B [6, 17] and EDIN-C [18]. The chromosomal gene encoding edin- $B$ is located within a pathogenicity island frequently associated with the $e t D$ gene encoding the exfoliative toxin ET-D [17]. EDIN-C is encoded by the pETB plasmid, which also carries genes encoding ET-B and conferring cadmium resistance [18].

A first epidemiological survey, involving staphylococcal strains isolated from patients hospitalized for various infectious diseases demonstrated a higher prevalence of edinencoding genes in this group compared to nasal strains isolated from healthy students [19]. Another study shows that edin-B is present in 7\% of bacteriemic S. aureus strains [17]. However, most other epidemiological data on edin are based on surveys focused on exfoliative toxins or PVL rather than EDIN toxin itself. For example, a genetic association between $e t D$ and $e d i n-B$ was detected in the emerging ST80 clone Panton-Valentine Leukocidin (PVL)-positive and community-acquired (CA) methicillin-resistant S. aureus (MRSA) [20]. This clone is spreading throughout France and Tunisia and is most frequently associated with infections of the skin and soft tissues. Also, two-thirds of the strains belonging to the emerging ST123 epidemic European fusidic acid-resistant impetigo clone 
1 (EEFIC) were positive for $e t B$ and sequence analysis of pETB2 (a close homolog of pETB) in 2 one of these strains suggested that it also bears edin- $C$ [21]. In this study, we have developed a PCR-based method, to detect EDIN isoforms 4 specifically. We demonstrate that $90 \%$ of all edin-bearing S. aureus isolates carry the type-C 5 allele. We also show that these isolates are more significantly associated with deep-seated soft 6 tissue infections than other types of infections (Fisher's exact test, $p=0.03$ ). 
S. aureus isolates

4

A total of 256 isolates of $S$. aureus belonging to the collection of the Bacteriology department of the Hospital University of Nice were analyzed. These isolates were recovered from randomly consecutive episodes of $S$. aureus infections in patients hospitalized during 2005. These isolates were obtained from various types of clinical samples, comprising blood cultures (28 bacteria); skin infections including chronic ulcers, burns or wounds ( 83 bacteria); urine samples (41 bacteria); sputum samples (69 bacteria); and various deep-seated soft tissue infections such as subcutaneous or visceral abscesses, spontaneously or post operative soft tissue infections (35 bacteria). For the last group, bacteria were isolated from specimens obtained by guidance radiography needle biopsy or during endoscopic and surgical procedures. All isolates were characterized using routine methods according to each manufacturer's recommendations. All were positive for catalase, DNAse production and mannitol fermentation in Chapman medium, and confirmed to be $S$. aureus by specific 32rapidStaph (BioMérieux, Marcy-l'Etoile, France).

Antibiotic susceptibility determinations

Antimicrobial susceptibility testing was performed on all isolates obtained during the study using the disk diffusion method [22] on Mueller-Hinton medium (Difco Laboratories, Detroit, MI) according to the recommendations of the French Antibiogram Committee [http://www.sfm.asso.fr/nouv/general.php?pa=2]. Antibiotics tested were penicillin G, oxacillin, erythromycin, clindamycin and fusidic acid to focus on epidemiologic profiles.

DNA isolation and PCR-based detection of genes

For edin detection, total DNA was isolated from bacterial strains grown overnight at $37^{\circ} \mathrm{C}$ in BHI medium. Bacteria were lysed in $10 \mathrm{mM}$ TrisHCl pH7.8, $100 \mathrm{mM} \mathrm{NaCl}, 1 \mathrm{mM}$ EDTA, $1 \%$ Triton X100. After incubation for 10 minutes at $100^{\circ} \mathrm{C}$, DNA was collected and frozen. PCR amplification was used to detect the presence of edin-A, B and C using the primers described in Table 1. We have determined optimized thermal cycling conditions for $e$ din-A (25 cycles of 
$195^{\circ} \mathrm{C}, 1 \mathrm{~min}$ annealing at $50^{\circ} \mathrm{C}$ and 1 min elongation at $\left.72^{\circ} \mathrm{C}\right)$ and $e$ din- $C(30$ cycles of $30 \mathrm{~s}$ at $294^{\circ} \mathrm{C}, 45 \mathrm{~s}$ annealing at $54^{\circ} \mathrm{C}$ and 1 min elongation at $72^{\circ} \mathrm{C}$ ). For the detection of other 3 virulence genes, total DNA was isolated from bacterial strains grown three hours at $37^{\circ} \mathrm{C}$ in 4 BHI medium. DNA was subsequently extracted with NucleoSpin Tissue (Macherey-Nagel $5 \mathrm{GmbH}$, Düren, Germany) according to manufacturer's recommendations. Briefly, bacteria 6 were pelleted by centrifugation at $8,000 \times g$ for $5 \mathrm{~min}$, resuspended in $180 \mu \mathrm{l}$ of lysis buffer 7 with $33 \mu \mathrm{l}$ of proteinase $\mathrm{K}(20 \mathrm{mg} / \mathrm{ml})$ (Invitrogen Life Technologies, Carlsbad, CA) and $3 \mu 1$ of 8 recombinant lysostaphin $(3 \mathrm{U} / \mu \mathrm{l})$ (Sigma-Aldrich, St Louis, MI), and incubated for $60 \mathrm{~min}$ at $9 \quad 37^{\circ} \mathrm{C}$. DNA samples were eluted with $100 \mu \mathrm{l}$ alkaline elution buffer (BE buffer, NucleoSpin Tissue, Macherey-Nagel). The presence of 30 genes, among the most prevalent virulenceassociated genes, was evaluated by PCR as described previously: staphylococcal enterotoxins (se) A, B, C, D, E, G, H, I, J, K, and Q, toxic shock syndrome toxin 1 (tst-1), exfoliative 13 toxins A, B and D (etA, etB, etD), PVL (lukS-PV-lukF-PV), LukDE leukocidin (lukE), nine 14 MSCRAMM (bbp, cna, ebpS, clfA, clfB, fib, fnbA, fnbB, eno). The accessory gene regulator 15 (agr) allele group was determined by multiplex PCR.

spa sequencing

spa typing was performed as described previously [23], using the spa typing website (http://www.spaserver.ridom.de/) that is developed by Ridom $\mathrm{GmbH}$ and curated by SeqNet.org (http://www.SeqNet.org/). Primers are indicated in Table 1.

Statistical analysis

The chi-square or Fisher's exact test for categorical variables was used to compare data as appropriate. A $P$ value of less than 0.05 was considered significant. 


\section{Results}

2

Detection of edin isoforms

S. aureus isolates analyzed in this study were collected at the university hospital of Nice from various infected patients. We designed primers with high sensitivity and specificity in order to detect, by PCR, edin-A, B and C alleles in these isolates. This was especially challenging for edin-C, which was poorly detected using a previously described pair of primers designed to amplify all edin isoforms. This is consistent with the fact that the sequence of edin- $C$ has the most substantial sequence variations in regions recognized by these primer sequences (17\% and 32\% homology with the forward and reverse primers, respectively) (Fig. 1) [19]. As shown in figure 1 , the three pairs of primers designed allowed us to amplify specifically a 455 bp DNA fragment for $e$ din- $A$ and $B$, and a 320 bp DNA fragment for $e d i n-C$.

We next analyze the 256 clinical isolates of $S$. aureus. We found that $14 \%$ (36) of these isolates were positive for one of the edin alleles. Among these 36 isolates, $90 \%$ were positive for edin-C and $5 \%$ were positive for either edin-A or $B$. To confirm the nature of the edin isoforms, we performed complete sequence analysis of five edin- $C$ encoding genes from randomly selected isolates. We also sequenced $e$ din- $A$ and $e$ din- $B$ encoding genes. These results confirm the specificity of the new primers used and demonstrate that edin- $C$ was more prevalent than other alleles of edin.

Detection of genes encoding virulence factors

The 36 isolates positive for edin genes were next analyzed for the distribution of major MSCRAMMs, various staphylococcal enterotoxins, exfoliative toxins, the toxic shock syndrome toxin 1 gene $t s t-1$, as well as leucotoxin family encoding genes. Among the staphylococcal MSCRAMM genes, eno, clfA and clfB were detected in all edin-positive isolates (Table 2). bbp, $f n b A$ and ebpS were the less frequently encountered MSCRAMMs among these isolates. However these adhesion factors had no preferential distribution among S. aureus isolated from various types of infections. Among the staphylococcal enterotoxins genes the most frequently encountered were seg, sei and sea (Table 2). In addition, $72 \%$ of edin-positive isolates (26 bacteria) contained a combination of these three genes. One edin-C bearing isolate, isolated from a urine sample, had only the sea enterotoxin gene. We detected the exfoliative toxin gene $e t D$ exclusively among the $e$ din- $B$ positive isolates. Two edin-A 
1 positive isolates carried the staphylococcal exfoliative gene et $B$. We found that 25 edin- $C$

2 positive $S$. aureus (78\%) were negative for $e t A$, etB and $e t D$ exfoliative toxin encoding genes.

3 We observed that only $22 \%$ of the edin-C positive $S$. aureus (7 out of 32 ) had at least one of

4 the two isoforms ( $e t A$ or $e t B$ ) of the exfoliative toxin gene. Five of these seven isolates carried both the $e t A$ and $e t B$ genes. No significant association was found between the presence of $t s t$ 1 , exfoliative toxins or leucotoxin family encoded genes and the types of infection. Finally, a large number of edin-positive $S$. aureus belonged to the agr group 1 (50\%, 18 bacteria), and to a lesser extent, to agr groups 2, 3 and 4 (17\%, 6 bacteria each) (Table 2).

spa-typing

Determination of the spa type of 26 edin-positive isolates [23] allowed us to exclude the clonal origin of all edin-C positive $S$. aureus in our survey. Among them, only six isolates could be classified as ST45 (two isolates), ST30 (two isolates), ST59 or ST26. The other 20 isolates showed a high variability of their spa type (Table 3). For 14 isolates, we determined new repeat sequences including unidentified 24-bp repeats thus defining new spa types. Among them, bacteria S7926 and S7262, isolated respectively in deep seated soft tissue and sputum sample from unrelated infected patients, presented the same spa type t6956 (Table 3). Together these data excluded the clonal origin of all edin- $C$ positive $S$. aureus in our survey.

Antibiotic susceptibility profiles

We next investigated whether edin-positive isolates were associated with specific antibiotic resistance profiles, such as community-acquired methicillin resistant $S$. aureus ST80 and fusidic acid-resistant impetigo clones [20, 21]. In relation with these studies, we determined the minimum inhibitory concentrations (MICs) of edin-positive isolates for the following antibiotics: penicillin G, methicillin, erythromycin, clindamycin and fusidic acid. Results were presented in Table 2. Only one isolate, positive for edin-C, showed a methicillinresistance. Finally, edin-positive isolates did not show any specific resistance profile to classical antibiotics used to cure infections by S. aureus (Table 2). 
1 Having shown no link between a high prevalence of edin- $C$ within our $S$. aureus isolates and 2 any phenotypic profile or clonal origin, we further analyzed the distribution of edin-positive 3 isolates with regard to the infectious sites. We noticed that isolates positive for edin- $C$ were 4 recovered at all sites of infection (Table 4). Strikingly, edin-positive S. aureus were more 5 significantly associated with deep-seated infections of soft tissues than other types of 6 infections $(25.7 \%$, Fischer exact test, $\mathrm{p}=0.03)$. No significant association was detected 7 between edin-positive or edin-negative isolates and other types of infections (blood, urine, 8 superficial soft tissue and sputum culture).

9 


\section{Discussion}

Our study shows that edin-positive $S$. aureus isolates are found in all types of clinical infections included in this study, with a global prevalence of $14 \%$. Moreover, we show that $90 \%$ of edin-positive isolates are positive for edin- $C$. This is consistent with a previous study performed with specific primers, which also reported a higher prevalence of $e$ din- $C$ in clinical isolates of S. aureus in Japan [24]. This points for the need of using specific primers to detect each edin isoform, especially edin- $C$, given its high prevalence. On the contrary, both studies point for a possible underestimation of the prevalence of edin-C in pathogenic $S$. aureus when consensus primers were used. Both the analysis of spa type and the distribution of various virulence factors among edin-positive $S$. aureus show their high genetic variability. Our results on the distribution of MSCRAMMs are consistent with previous findings [25]. Classically, edin and exfoliative toxin encoding genes are associated in specific lineages responsible for skin infections [17, 21, 24]. Interestingly, here we show that edin- $C$ is not strictly associated with genes encoding exfoliative toxin of serotypes A/B/D (7 bacteria edin$C$-positive and $e t$-positive, versus 25 bacteria $e d i n$ - $C$-positive and $e t$-negative). The high prevalence of edin-C-positive and et-negative isolates observed in our study might be explained by the plasticity of the pETB plasmid, which has been previously reported in two different variants $[18,21]$. Also, a recent study shows genetic variations in pathogenicity islands encoding EDIN-B [17].

Given that spa analysis constitutes a good tool for epidemiological typing of $S$. aureus [26], the use of this method allowed us to exclude the clonal origin of edin- $C$ positive isolates. The fact that edin- $C$ is plasmid born might explain its presence in isolates of various genetic backgrounds.

S. aureus positive for edin are more frequently associated with deep-seated infections of soft tissues. We have previously established that infection of endothelial cells, and other cell types, by EDIN-producing S. aureus triggers the formation of transcellular tunnels named macroapertures $[12,13]$. Opening of transcellular tunnels in the endothelium suggested that EDIN might favour bacterial extravasation in tissues during bacteremia. In a mouse model of intravascular injection of $S$. aureus, we have observed that EDIN plays no detectable role in the persistence of bacteria in the blood stream [15]. This data is consistent with the absence of a higher prevalence of edin positive $S$. aureus recovered from patients associated bacteriemia in this study. In contrast, in this model of mouse infection EDIN toxin promotes formation of infection foci [15]. This suggested that EDIN might enhance the invasive capacity of $S$. 
1 aureus. The hypothesis of a role of EDIN in $S$. aureus infection is also supported by our 2 present findings showing that $S$. aureus associated with deep-seated infections of soft tissues 3 have a higher prevalence of edin. However, whether or not edin-positive S. aureus is 4 preferentially associated with a specific type of deep seated infection remains to be further 5 determined".

6

7 Acknowledgments We are grateful to Fernand Girard-Pipau and Claire Poyart for providing 8 various strains of $S$. aureus and Pr Jean-Louis Mege for critical reading of the manuscript.

9 Our laboratory is supported by an institutional funding from the INSERM, a grant from 10 the Agence Nationale de la Recherche (ANR RPV07055ASA) and the Association pour la 11 Recherche sur le Cancer (ARC 4906). 


\section{References}

2

3 [1] Lowy FD (1998) Staphylococcus aureus infections. N Engl J Med 339 (8):520-532

4 [2] Fournier B, Philpott DJ (2005) Recognition of Staphylococcus aureus by the innate 5 immune system. Clin Microbiol Rev 18 (3):521-540

6 [3] Becker K, Friedrich AW, Lubritz G et al (2003) Prevalence of genes encoding 7 pyrogenic toxin superantigens and exfoliative toxins among strains of Staphylococcus aureus isolated from blood and nasal specimens. J Clin Microbiol 41 (4):1434-1439

[4] Boquet P, Lemichez E (2003) Bacterial virulence factors targeting Rho GTPases: parasitism or symbiosis? Trends Cell Biol 13 (5):238-246

[5] Dinges MM, Orwin PM, Schlievert PM (2000) Exotoxins of Staphylococcus aureus. Clin Microbiol Rev 13 (1):16-34

[6] Wilde C, Aktories K (2001) The Rho-ADP-ribosylating C3 exoenzyme from Clostridium botulinum and related C3-like transferases. Toxicon 39 (11):1647-1660

[7] Wilde C, Vogelsgesang M, Aktories K (2003) Rho-specific Bacillus cereus ADPribosyltransferase C3cer cloning and characterization. Biochemistry 42 (32):9694-9702

[8] Chardin P, Boquet P, Madaule P et al (1989) The mammalian G protein rhoC is ADPribosylated by Clostridium botulinum exoenzyme $\mathrm{C} 3$ and affects actin microfilaments in Vero cells. EMBO J 8 (4):1087-1092

[9] Aktories K, Barbieri JT (2005) Bacterial cytotoxins: targeting eukaryotic switches. Nat Rev Microbiol 3 (5):397-410

[10] Jaffe AB, Hall A (2005) Rho GTPases: biochemistry and biology. Ann Rev Cell Dev Biol 21:247-269

[11] Visvikis O, Maddugoda MP, Lemichez E (2010) Direct modifications of Rho proteins: deconstructing GTPase regulation. Biol Cell 102 (7):377-389

[12] Boyer L, Doye A, Rolando M et al (2006) Induction of transient macroapertures in endothelial cells through RhoA inhibition by Staphylococcus aureus factors. J Cell Biol 173 (5):809-819

[13] Lemichez E, Lecuit M, Nassif X et al (2010) Breaking the wall: targeting of the endothelium by pathogenic bacteria. Nat Rev Microbiol 8 (2):93-104

[14] Rolando M, Munro P, Stefani Cet al (2009) Injection of Staphylococcus aureus EDIN by the Bacillus anthracis protective antigen machinery induces vascular permeability. Infect Immun 77 (9):3596-3601 
1 [15] Munro P, Benchetrit M, Nahori MA et al (2010) Staphylococcus aureus EDIN toxin 2 promotes formation of indection foci in a mouse model of bacteremia. Infect Immun 78 3 (8):3404-3411

4 [16] Inoue S, Sugai M, Murooka Y et al (1991) Molecular cloning and sequencing of the epidermal cell differentiation inhibitor gene from Staphylococcus aureus. Biochem biophys Research Com 174 (2):459-464

[17] Franke GC, Bockenholt A, Sugai M et al (2009) Epidemiology, variable genetic organisation and regulation of the EDIN-B toxin in Staphylococcus aureus from bacteraemic patients. Microbiology 156 (3):860-872.

[18] Yamaguchi T, Hayashi T, Takami H et al (2001) Complete nucleotide sequence of a Staphylococcus aureus exfoliative toxin B plasmid and identification of a novel ADPribosyltransferase, EDIN-C. Infect Immun 69 (12):7760-7771

[19] Czech A, Yamaguchi T, Bader L et al (2001) Prevalence of Rho-inactivating epidermal cell differentiation inhibitor toxins in clinical Staphylococcus aureus isolates. J Infect Dis 184 (6):785-788

[20] Ben Nejma M, Mastouri M, Bel Hadj Jrad B et al (2008) Characterization of ST80 Panton-Valentine leukocidin-positive community-acquired methicillin-resistant Staphylococcus aureus clone in Tunisia. Diagn Microbiol Infectious Dis In press [Epub ahaed of print]

[21] O'Neill AJ, Larsen AR, Skov R, Henriksen AS, Chopra I (2007) Characterization of the epidemic European fusidic acid-resistant impetigo clone of Staphylococcus aureus. J Clin Microbiol 45 (5):1505-1510

[22] Bauer AW, Kirby WM, Sherris JC et al (1966) Antibiotic susceptibility testing by a standardized single disk method. Am J Clin Pathol 45 (4):493-496

[23] Harmsen D, Claus H, Witte W et al (2003) Typing of methicillin-resistant Staphylococcus aureus in a university hospital setting by using novel software for spa repeat determination and database management. J Clin Microbiol 41 (12):5442-5448 [24] Yamaguchi T, Yokota Y, Terajima J et al (2002) Clonal association of Staphylococcus aureus causing bullous impetigo and the emergence of new methicillin-resistant clonal groups in Kansai district in Japan. J Infect Dis 185 (10):1511-1516 
1 [26] Petersson AC, Olsson-Liljequist B, Miorner H, et al (2010) Evaluating the usefulness

2 of spa typing, in comparison with pulsed-field gel electrophoresis, for epidemiological typing

3 of methicillin-resistant Staphylococcus aureus in a low-prevalence region in Sweden 2000-

4 2004. Clin Microbiol Infect 16 (5):456-462

5 [27] Holtfreter S, Bauer K, Thomas D et al (2004) egc-Encoded superantigens from

6 Staphylococcus aureus are neutralized by human sera much less efficiently than are classical

7 staphylococcal enterotoxins or toxic shock syndrome toxin. Infect Immun 72 (7):4061-4071

8 [28] Johnson WM, Tyler SD, Ewan EP et al (1991) Detection of genes for enterotoxins,

9 exfoliative toxins, and toxic shock syndrome toxin 1 in Staphylococcus aureus by the 10 polymerase chain reaction. J Clin Microbiol 29 (3):426-430

11 [29] Jarraud S, Mougel C, Thioulouse J et al (2002) Relationships between Staphylococcus 12 aureus genetic background, virulence factors, agr groups (alleles), and human disease. Infect 13 Immun 70 (2):631-641

14 [30] Lina G, Boutite F, Tristan A et al (2003) Bacterial competition for human nasal cavity 15 colonization: role of Staphylococcal agr alleles. Appl Environmental Microbiol 69 (1):18-23 


\section{$1 \quad$ Figure legend}

2

3 Fig. 1 Characterization of the three edin alleles. A) PCR amplification of edin- $A$, edin- $B$ and

4 edin-C from $S$. aureus strains S25edin-A(+)[15], S7256edin-B(+) and S7475edin-C(+) (this 5 study), respectively, using specific oligonucleotides edinA, edinB, edinC (Table 1) and the 6 previously described edin oligonucleotides referred as edinX [19]. B) Sequence alignments of 7 edin- $A$, edin- $B$ and edin- $C$ showing sequence homologies and localization of each 8 oligonucleotide (underlined: $e \operatorname{din} X$; highlighted: $e \operatorname{din} A, e \operatorname{din} B$ and $e \operatorname{din} C$ ).

9

10

11

12

13

14

15

16

17

18

19

20

21

22

23

24

25 
1 Table 1: Oligonucleotides primers used in this study

2

\begin{tabular}{|c|c|c|c|}
\hline Gene & Primer sequences & $\begin{array}{l}\text { Size } \\
(\mathrm{kb})\end{array}$ & References \\
\hline \multirow[t]{2}{*}{$\operatorname{edin} \mathrm{A}$} & Sense 5'-GGAGATATTAATAAGCTAGATTC-3' & 455 & This study \\
\hline & Antisense 5'-ATTTTCTTTTTATCATTTGACAATTCT-3' & & \\
\hline \multirow[t]{2}{*}{ edin $\mathrm{B}$} & Sense 5'-GGTGACGTGAACAAATTATCCGA-3' & 455 & This study \\
\hline & Antisense 5'-ATCTTTCTTTTGTTATCAGAAAGTTTA-3' & & \\
\hline \multirow[t]{2}{*}{ edin $\mathrm{C}$} & Sense 5'-CGCCATTAAGGTCTAGTCAAGG-3' & 320 & This study \\
\hline & Antisense 5'-TAGGTCTTCCAGCTAATGCAGC-3' & & \\
\hline \multirow[t]{2}{*}{$b b p$} & Sense 5'-AACTACATCTAGTACTCAACAACAG-3' & 575 & {$[25]$} \\
\hline & Antisense 5'-ATGTGCTTGAATAACACCATCATCT-3' & & \\
\hline \multirow[t]{2}{*}{ cna } & Sense 5'-GTCAAGCAGTTATTAACACCAGAC-3' & 423 & [25] \\
\hline & Antisense 5'-AATCAGTAATTGCACTTTGTCCACTG-3' & & \\
\hline \multirow[t]{2}{*}{$e b p S$} & Sense 5'-CATCCAGAACCAATCGAAGAC-3' & 186 & [25] \\
\hline & Antisense 5'-CTTAACAGTTACATCATCATGTTTATCTTTG-3' & & \\
\hline \multirow[t]{2}{*}{ fnbA } & Sense 5'-GTGAAGTTTTAGAAGGTGGAAAGATTAG -3' & 643 & [25] \\
\hline & Antisense 5'-GCTCTTGTAAGACCATTTTTCTTCAC-3' & & \\
\hline \multirow[t]{2}{*}{$f n b B$} & Sense 5'-GTAACAGCTAATGGTCGAATTGATACT-3' & 524 & {$[25]$} \\
\hline & Antisense 5'-CAAGTTCGATAGGAGTACTATGTTC-3' & & \\
\hline \multirow[t]{2}{*}{ fib } & Sense 5'-CTACAACTACAATTGCCGTCAACAG-3' & 404 & [25] \\
\hline & Antisense 5'-GCTCTTGTAAGACCATTTTCTTCAC-3' & & \\
\hline \multirow[t]{2}{*}{ clfA } & Sense 5'-ATTGGCGTGGCTTCAGTGCT-3' & 292 & [25] \\
\hline & Antisense 5'-CGTTTCTTCCGTAGTTGCATTTG-3' & & \\
\hline \multirow[t]{2}{*}{ clfB } & Sense 5'-ACATCAGTAATAGTAGGGGGCAAC-3' & 205 & [25] \\
\hline & Antisense 5'-TTCGCACTGTTTGTGTTTGCAC-3' & & \\
\hline \multirow[t]{2}{*}{ eno } & Sense 5'- ACGTGCAGCAGCTGACT-3' & 302 & [25] \\
\hline & Antisense 5'- CAACAGCATYCTTCAGTACCTTC-3' & & \\
\hline \multirow[t]{2}{*}{ sea } & Sense 5'-GCAGGGAACAGCTTTAGGC-3' & 520 & {$[27]$} \\
\hline & Antisense 5'-GTTCTGTAGAAGTATGAAACACG-3' & & \\
\hline \multirow[t]{2}{*}{ seb } & Sense 5'-ATGTAATTTTGATATTCGCAGTG-3' & 683 & {$[27]$} \\
\hline & Antisense 5'-TGCAGGCATCATATCATACCA-3' & & \\
\hline sec & Sense 5'-CTTGTATGTATGGAGGAATAACAA-3' & 283 & {$[27]$} \\
\hline
\end{tabular}




\begin{tabular}{|c|c|c|c|}
\hline & Antisense 5'-TGCAGGCATCATATCATACCA-3' & & \\
\hline \multirow[t]{2}{*}{ sed } & Sense 5'-GTGGTGAAATAGATAGGACTGC-3' & 384 & [27] \\
\hline & Antisense 5'-ATATGAAGGTGCTCTGTGG-3' & & \\
\hline \multirow[t]{2}{*}{ see } & Sense 5'-TACCAATTAACTTGTGGATAGAC-3' & 170 & [27] \\
\hline & Antisense 5'-CTCTTTGCACCTTACCGC-3' & & \\
\hline \multirow[t]{2}{*}{$\operatorname{seg}$} & Sense 5'-CGTCTCCACCTGTTGAAGG-3' & 327 & [27] \\
\hline & Antisense 5'-CCAAGTGATTGTCTATTGTCG-3' & & \\
\hline \multirow[t]{2}{*}{$\operatorname{seh}$} & Sense 5'-CAACTGCTGATTTAGCTCAG-3' & 360 & [27] \\
\hline & Antisense 5'-GTCGAATGAGTAATCTCTAGG-3' & & \\
\hline \multirow[t]{2}{*}{ sei } & Sense 5'-CAACTCGAATTTTCAACAGGTAC-3' & 465 & [27] \\
\hline & Antisense 5'- CAGGCAGTCCATCTCCTG-3' & & \\
\hline \multirow[t]{2}{*}{$\operatorname{sej}$} & Sense 5'- CATCAGAACTGTTGTTCCGCTAG-3' & 142 & {$[27]$} \\
\hline & Antisense 5'- CTGAATTTTACCATCAAAGGTAC-3' & & \\
\hline \multirow[t]{2}{*}{ sek } & Sense 5'- ATGGCGGAGTCACAGCTACT-3' & 197 & [27] \\
\hline & Antisense 5'-TGCCGTTATGTCCATAAATGTT-3' & & \\
\hline \multirow[t]{2}{*}{$\operatorname{seq}$} & Sense 5'-GAACCTGAAAAGCTTCAAGGA-3' & 209 & [27] \\
\hline & Antisense 5'-ATTCGCCAACGTAATTCCAC-3' & & \\
\hline \multirow[t]{2}{*}{ eta } & Sense 5'-CTAGTGCATTTGTTATTCAA-3' & 119 & [28] \\
\hline & Antisense 5'-TGCATTGACACCATAGTACT-3' & & \\
\hline \multirow[t]{2}{*}{$e t b$} & Sense 5'-ACGGCTATATACATTCAATT-3' & 200 & [28] \\
\hline & Antisense 5'-TCCATCGATAATATACCTAA-3' & & \\
\hline \multirow[t]{2}{*}{ etd } & Sense 5'-ATGACTAAAAATATATTAAAAAGTT-3' & 846 & This study \\
\hline & Antisense 5'-CTAATGAGACTGTAATTCAGC-3’ & & \\
\hline \multirow[t]{2}{*}{$l u k P V$} & Sense 5'-ATCATTAGGTAAAATGTCTGGACATGATCCA-3' & 433 & [29] \\
\hline & Antisense 5'-GCATCAASTGTATTGGATAGCAAAAGC-3' & & \\
\hline \multirow[t]{2}{*}{ lukE } & Sense 5'-TGAAAAAGGTTCAAAGTTGATACGAG-3' & 269 & [29] \\
\hline & Antisense 5'-TGTATTCGATAGCAAAAGCAGTGCA-3' & & \\
\hline \multirow[t]{2}{*}{ tst-1 } & Sense 5'-GCTTGCGACAACTGCTACAG-3' & 559 & [27] \\
\hline & Antisense 5'-TGGATCCGTCATTCATTGTTAA-3' & & \\
\hline \multirow[t]{2}{*}{ agrl } & Sense 5'-ATGCACATGG TGCACATGC-3' & 439 & [30] \\
\hline & Antisense 5'-GTCACAAGTACTATAAGCTGCGAT-3' & & \\
\hline \multirow[t]{2}{*}{ agr2 } & Sense 5'-ATGCACATGG TGCACATGC-3' & 572 & [30] \\
\hline & Antisense 5'-TATTACTAATTGAAAAGTGC CATAGC-3' & & \\
\hline
\end{tabular}




\begin{tabular}{|llcc|}
\hline agr3 & Sense 5'-ATGCACATGG TGCACATGC-3' & 321 & [30] \\
& Antisense 5'-GTAATGTAATAGCTTGTATAATAATACCCAG-3' & & \\
\multirow{2}{*}{ agr4 } & Sense 5'-ATGCACATGG TGCACATGC-3' & 657 & {$[30]$} \\
& Antisense 5'-CGATAATGCCGTAATACCCG-3' & & \\
spa & Sense5'-TGTAAAACGACGGCCAGTTAAAGACGATCCTTCGGTGAGC-3' & [23] \\
& Antisense5' 'CAGGAAACAGCTATGACCCAGCAGTAGTGCCGTTTGCTT-3'
\end{tabular}

1 
Table 2: Virulence profile and antibiotic susceptibility of clinical edin-positive $S$. aureus isolates.

\begin{tabular}{|c|c|c|c|c|c|c|c|c|c|c|c|c|}
\hline & \multicolumn{2}{|c|}{ blood $(n=2)$} & \multicolumn{2}{|c|}{$\begin{array}{l}\text { superficial soft tissue } \\
(n=11)\end{array}$} & \multicolumn{2}{|c|}{ urine sample $(n=4)$} & \multicolumn{2}{|c|}{ sputum sample $(n=10)$} & \multicolumn{2}{|c|}{$\begin{array}{c}\text { deep-seated soft tissue } \\
(n=9)\end{array}$} & \multicolumn{2}{|c|}{ total $(n=36)$} \\
\hline & $\mathrm{n}$ & $\%$ & $\mathrm{n}$ & $\%$ & $\mathrm{n}$ & $\%$ & $\mathrm{n}$ & $\%$ & $\mathrm{n}$ & $\%$ & $\mathrm{n}$ & $\%$ \\
\hline \multicolumn{13}{|c|}{$\begin{array}{l}\text { Virulence profile } \\
\text { MSCRAMMs }\end{array}$} \\
\hline$b b p$ & 0 & 0 & 3 & 27 & 3 & 75 & 3 & 30 & 6 & 67 & 15 & 42 \\
\hline cna & 1 & 50 & 9 & 82 & 4 & 100 & 10 & 100 & 8 & 89 & 32 & 89 \\
\hline ebps & 0 & 0 & 5 & 45 & 1 & 25 & 6 & 60 & 6 & 67 & 18 & 50 \\
\hline$f n b A$ & 1 & 50 & 7 & 64 & 1 & 25 & 5 & 50 & 5 & 56 & 19 & 53 \\
\hline$f n b B$ & 1 & 50 & 11 & 100 & 4 & 100 & 7 & 70 & 8 & 89 & 31 & 86 \\
\hline fib & 2 & 100 & 10 & 91 & 4 & 100 & 7 & 70 & 9 & 100 & 32 & 89 \\
\hline ClfA & 2 & 100 & 11 & 100 & 4 & 100 & 10 & 100 & 9 & 100 & 36 & 100 \\
\hline$C l f B$ & 2 & 100 & 11 & 100 & 4 & 100 & 10 & 100 & 9 & 100 & 36 & 100 \\
\hline eno & 2 & 100 & 11 & 100 & 4 & 100 & 10 & 100 & 9 & 100 & 36 & 100 \\
\hline \multicolumn{13}{|l|}{ SEs } \\
\hline sea & 1 & 50 & 10 & 91 & 3 & 75 & 9 & 90 & 9 & 100 & 32 & 89 \\
\hline seb & 1 & 50 & 7 & 64 & 2 & 50 & 8 & 80 & 6 & 67 & 24 & 67 \\
\hline $\sec$ & 1 & 50 & 7 & 64 & 2 & 50 & 10 & 100 & 6 & 67 & 26 & 72 \\
\hline sed & 0 & 0 & 8 & 73 & 3 & 75 & 6 & 60 & 6 & 67 & 23 & 64 \\
\hline see & 0 & 0 & 0 & 0 & 0 & 0 & 0 & 0 & 0 & 0 & 0 & 0 \\
\hline sek & 1 & 50 & 5 & 45 & 1 & 25 & 7 & 70 & 2 & 22 & 16 & 44 \\
\hline seq & 1 & 50 & 3 & 27 & 1 & 25 & 5 & 50 & 5 & 56 & 15 & 42 \\
\hline seg & 2 & 100 & 10 & 91 & 2 & 50 & 10 & 100 & 9 & 100 & 33 & 92 \\
\hline seh & 0 & 0 & 0 & 0 & 0 & 0 & 0 & 0 & 1 & 11 & 1 & 3 \\
\hline sei & 2 & 100 & 11 & 100 & 2 & 50 & 9 & 90 & 8 & 89 & 32 & 89 \\
\hline sej & 0 & 0 & 1 & 9 & 0 & 0 & 2 & 20 & 1 & 11 & 4 & 11 \\
\hline$t s t-1$ & 2 & 100 & 6 & 55 & 1 & 25 & 7 & 70 & 5 & 56 & 21 & 58 \\
\hline etA & 0 & 0 & 2 & 18 & 0 & 0 & 2 & 20 & 2 & 22 & 6 & 17 \\
\hline etB & $1^{\text {\& }}$ & 50 & $4^{\$}$ & 36 & 0 & 0 & 2 & 20 & 1 & 11 & 8 & 22 \\
\hline etD & 0 & 0 & 0 & 0 & $1^{*}$ & 25 & $1^{*}$ & 10 & 0 & 0 & 2 & 6 \\
\hline lukPV & 0 & 0 & 1 & 9 & 1 & 25 & 0 & 0 & 3 & 33 & 5 & 14 \\
\hline lukE & 2 & 100 & 8 & 73 & 3 & 75 & 5 & 50 & 4 & 44 & 24 & 67 \\
\hline \multicolumn{13}{|l|}{ agr group } \\
\hline agr1 & 1 & 50 & 3 & 27 & 3 & 75 & 6 & 60 & 5 & 56 & 18 & 50 \\
\hline agr2 & 1 & 50 & 3 & 27 & 1 & 25 & 1 & 10 & 0 & 0 & 6 & 17 \\
\hline agr3 & 0 & 0 & 3 & 27 & 0 & 0 & 0 & 0 & 3 & 33 & 6 & 17 \\
\hline agr4 & 0 & 0 & 2 & 18 & 0 & 0 & 3 & 30 & 1 & 11 & 6 & 17 \\
\hline \multicolumn{13}{|c|}{ antibiotic resistance profile } \\
\hline penicillin $G$ & 1 & 50 & 5 & 45 & 2 & 50 & 8 & 80 & 6 & 67 & 22 & 61 \\
\hline Methicillin & 0 & 0 & 0 & 0 & 0 & 0 & 0 & 0 & 1 & 11 & 1 & 3 \\
\hline Erythromycin & 0 & 0 & 1 & 9 & 0 & 0 & 1 & 10 & 1 & 11 & 3 & 8 \\
\hline Clindamycin & 0 & 0 & 0 & 0 & 0 & 0 & 0 & 0 & 1 & 11 & 1 & 3 \\
\hline Fusidic acid & 0 & 0 & 0 & 0 & $1 a$ & 25 & 0 & 0 & $1 \mathrm{a}$ & 11 & 2 & 6 \\
\hline
\end{tabular}


Table 3 : spa-type of 36 edin-positive Staphylococcus aureus isolates.

\begin{tabular}{|c|c|c|c|}
\hline Strains & EDIN type & Spa-type or repeat sequences & MLST \\
\hline \multicolumn{4}{|l|}{ Blood } \\
\hline $\mathrm{S} 25$ & $\mathrm{~A}$ & t6403 & - \\
\hline S7232 & $\mathrm{C}$ & $\mathrm{NT}^{\$}$ & - \\
\hline \multicolumn{4}{|c|}{ Deep-seated soft tissue } \\
\hline S7272 & $\mathrm{C}$ & t6953 & - \\
\hline S7404 & $\mathrm{C}$ & t6649 & - \\
\hline S7408 & $\mathrm{C}$ & t6484 & - \\
\hline S7466 & $\mathrm{C}$ & t012 & ST-30 \\
\hline S7595 & $\mathrm{C}$ & NT & - \\
\hline S7600 & $\mathrm{C}$ & t6677 & - \\
\hline S7926 & $\mathrm{C}$ & t6956 & - \\
\hline S8028* & $\mathrm{C}$ & NT & - \\
\hline S8087 & $\mathrm{C}$ & t6481 & - \\
\hline \multicolumn{4}{|c|}{ Sputum sample } \\
\hline S7225 & $\mathrm{C}$ & $\mathrm{t} 2726$ & - \\
\hline S7259 & $\mathrm{C}$ & $\mathrm{t} 2088$ & - \\
\hline S7262 & $\mathrm{C}$ & t6956 & - \\
\hline S7413 & $\mathrm{C}$ & t6483 & - \\
\hline S7535 & $\mathrm{C}$ & $\mathrm{t} 2647$ & - \\
\hline S7634 & $\mathrm{C}$ & t6954 & - \\
\hline S7649 & $\mathrm{B}$ & NT & - \\
\hline S7920 & $\mathrm{C}$ & NT & - \\
\hline S7965 & $\mathrm{C}$ & NT & - \\
\hline S8100 & $\mathrm{C}$ & t015 & ST-45 \\
\hline \multicolumn{4}{|c|}{ Superficial soft tissue } \\
\hline S7181 & $\mathrm{C}$ & t6650 & - \\
\hline S7183 & $\mathrm{C}$ & NT & - \\
\hline S7436 & $\mathrm{C}$ & t6957 & - \\
\hline S7475 & $\mathrm{C}$ & t6480 & - \\
\hline S7526 & $\mathrm{C}$ & $\mathrm{t} 137$ & - \\
\hline
\end{tabular}




\begin{tabular}{|c|c|c|c|}
\hline S7539 & C & NT & - \\
\hline S7569 & A & t031 & ST-45 \\
\hline S7599 & C & t6482 & - \\
\hline S7932 & C & NT & - \\
\hline S7938 & C & t620 & - \\
\hline S7977 & C & t078 & - \\
\hline Urine sample & t012 & ST-26 \\
\hline S7256 & B & t645 & ST-30 \\
\hline S7322 & C & t216 & ST-59 \\
\hline S7906 & C & C &
\end{tabular}

* MRSA bacteria

$\$$ Not typable 
Table 4: Presence of edin genes in 256 Staphylococcus aureus isolates associated with various clinical syndromes.

\begin{tabular}{lcrccc}
\hline \multirow{2}{*}{ Source of isolates (N) } & \multicolumn{2}{c}{ Number of edin } & \multicolumn{3}{c}{ edin-isoforms } \\
\cline { 3 - 5 } & isolates & (\%) & edinA & edinB & edinC \\
\hline Blood (28) & $2(7.1)$ & 1 & 0 & 1 \\
Urine (41) & $4(9.8)$ & 0 & 1 & 3 \\
Superficial soft tissue (83) & $11(13.3)$ & 1 & 0 & 10 \\
Deep-seated soft tissue (35) & $9(25.7)^{*}$ & 0 & 0 & 9 \\
Sputum (69) & $10(14.5)$ & 0 & 1 & 9 \\
Total (256) & $\mathbf{3 6 ( 1 4 )}$ & $\mathbf{2 ( 5 )}$ & $\mathbf{2 ( 5 )}$ & $\mathbf{3 2}(\mathbf{5 0})$ \\
\hline
\end{tabular}

$* \mathrm{p}<0,05$ (Fisher's exact test) 
High prevalence of edin- $C$ encoding RhoA-targeting toxin in clinical strains of Staphylococcus aureus

Patrick Munro $^{1}$, René Clément ${ }^{1}$, Jean-Philippe Lavigne ${ }^{4,5}$, Céline Pulcini ${ }^{2,3}$, Emmanuel Lemichez $^{1}$ and Luce Landraud ${ }^{1,6^{*}}$

Running title: EDIN exotoxins in S. aureus infections

1 INSERM, U895, C3M, toxines microbiennes dans la relation hôte pathogènes, Université de Nice-Sophia-Antipolis, UFR Médecine, IFR50, Nice, F-06204, France

2 Université de Nice-Sophia-Antipolis, UFR Médecine, IFR50, Nice, F-06204, France

3 Service d'Infectiologie, Hôpital l'Archet 1, Route Saint Antoine de Ginestière, BP 3079, 06202 Nice Cedex 3, France

4 INSERM, Espri 26, Université Montpellier 1, UFR de Médecine, Nîmes, France

5 Laboratoire de Bactériologie, CHU Caremeau, Nîmes, France

6 Laboratoire de Bactériologie, CHU de Nice, Hôpital l'Archet, Nice, France.

\section{* Corresponding author:}

Luce Landraud, INSERM, U895, C3M, toxines microbiennes dans la relation hôte pathogènes, Université de Nice-Sophia-Antipolis, UFR Médecine, IFR50, Nice, F-06204, France, and Laboratoire de Bactériologie, CHU de Nice, Hôpital l'Archet, Nice, France.

Telephone: 0033489064261

Fax: 0033489064260

Mail: landraud.1@chu-nice.fr

Abstract word count: 173 
Abstract Staphylococcus aureus, a major causative agent of human infection produces a large array of virulence factor including various toxins. Among them, the host RhoA GTPase targeting EDIN toxins are considered as potential virulence factors. Using the polymerase chain reaction, we analyzed the virulence profile of $256 \mathrm{~S}$. aureus strains isolated from various clinical sites of infections. We developed specific primers to detect the three isoforms of edin encoding genes. We found a prevalence of 14\% (36 strains) of edin encoding genes among these clinical strains. Strikingly, we found that $90 \%$ of all edin-bearing S. aureus strains carried the type-C allele. Both the spa types and the profile of virulence factors of these edin-positive strains are highly variable. Notably, we show for the first time that edin-C positive strains were more frequently recovered from deep-seated infections than other types of infections. Our present work thus strongly suggests that presence of $e$ din- $C$ is a risk factor of $S$. aureus dissemination in tissues and thus represents a predictive marker for a pejorative evolution of staphylococcal infections.

\section{Keywords}

Staphylococcus aureus, EDIN, toxin, ADP-ribosyltransferase, virulence factors, Rho GTPases. 


\section{Introduction}

Staphylococcus aureus is a common bacterium, which is responsible for a unique variety of infections [1]. Development of pejorative forms of staphylococcal infections involves the combined action of numerous bacterial virulence factors, which corrupt host responses [2]. Bacterial virulence factors include specific adhesins, collectively referred as Microbial Surface Components Recognizing endothelial cell Adhesive Matrix Molecules (MSCRAMMs) and a large variety of toxins, such as the exfoliative toxins (ETs), hemolysin, leukocidin, enterotoxins and EDINs (epidermal cell differentiation inhibitors) [3-6].

EDINs belong to the family of Clostridium botulinum C3 exoenzyme [6, 7]. They are members of a group of major bacterial virulence factors targeting host Rho GTPases [4, 6-9]. Rho proteins control essential cellular processes such as cell polarity, movement and phagocytosis, as well as cohesion of intercellular junctions [6, 10, 11]. Recent findings suggest that EDINs might favor bacterial dissemination in tissues, by a haematogenous route, through induction of large transcellular tunnels in endothelial cells named macroapertures [12-14]. Indeed, recent data show that $S$. aureus EDIN toxin promotes formation of infection foci in a mouse model of bacteremia [15]. To date, three isoforms of EDIN have been characterized. These comprise the first discovered EDIN isoform (EDIN-A), isolated from the E-1 strain of S. aureus [16], as well as EDIN-B [6, 17] and EDIN-C [18]. The chromosomal gene encoding edin- $B$ is located within a pathogenicity island frequently associated with the etD gene encoding the exfoliative toxin ET-D [17]. EDIN-C is encoded by the pETB plasmid, which also carries genes encoding ET-B and conferring cadmium resistance [18].

A first epidemiological survey, involving staphylococcal strains isolated from patients hospitalized for various infectious diseases demonstrated a higher prevalence of edinencoding genes in this group compared to nasal strains isolated from healthy students [19]. Another study shows that edin-B is present in $7 \%$ of bacteriemic $S$. aureus strains [17]. However, most other epidemiological data on edin are based on surveys focused on exfoliative toxins or PVL rather than EDIN toxin itself. For example, a genetic association between $e t D$ and $e d i n-B$ was detected in the emerging ST80 clone Panton-Valentine Leukocidin (PVL)-positive and community-acquired (CA) methicillin-resistant S. aureus (MRSA) [20]. This clone is spreading throughout France and Tunisia and is most frequently associated with infections of the skin and soft tissues. Also, two-thirds of the strains belonging to the emerging ST123 epidemic European fusidic acid-resistant impetigo clone 
(EEFIC) were positive for $e t B$ and sequence analysis of pETB2 (a close homolog of pETB) in one of these strains suggested that it also bears edin- $C$ [21].

In this study, we have developed a PCR-based method, to detect EDIN isoforms specifically. We demonstrate that $90 \%$ of all edin-bearing S. aureus strains carry the type-C allele. We also show that these strains are more significantly associated with deep-seated soft tissue infections than other types of infections (Fisher's exact test, $\mathrm{p}=0.03$ ). 


\section{Materials and methods}

S. aureus isolates

A total of 256 strains of $S$. aureus were retrospectively collected from patients hospitalized at the university hospital of Nice during 2005. These strains were obtained from various types of clinical samples, comprising blood cultures (28 strains); skin infections including chronic ulcers, burns or wounds (83 strains); urine samples (41 strains); sputum samples (69 strains); and various deep-seated soft tissue infections such as subcutaneous or visceral abscesses, spontaneously or post operative soft tissue infections (35 strains). All isolates were characterized using routine methods according to each manufacturer's recommendations. All were positive for catalase, DNAse production and mannitol fermentation in Chapman medium, and confirmed to be $S$. aureus by specific 32rapidStaph (BioMérieux, Marcyl'Etoile, France).

Antibiotic susceptibility determinations

Antimicrobial susceptibility testing was performed on all isolates obtained during the study using the disk diffusion method [22] on Mueller-Hinton medium (Difco Laboratories, Detroit, MI) according to the recommendations of the French Antibiogram Committee [http://www.sfm.asso.fr/nouv/general.php?pa=2]. Antibiotics tested were penicillin G, oxacillin, erythromycin, clindamycin and fusidic acid to focus on epidemiologic profiles.

DNA isolation and PCR-based detection of genes

For edin detection, total DNA was isolated from bacterial strains grown overnight at $37^{\circ} \mathrm{C}$ in BHI medium. Bacteria were lysed in $10 \mathrm{mM}$ TrisHCl pH7.8, $100 \mathrm{mM} \mathrm{NaCl}, 1 \mathrm{mM}$ EDTA, $1 \%$ Triton X100. After incubation for 10 minutes at $100^{\circ} \mathrm{C}$, DNA was collected and frozen. PCR amplification was used to detect the presence of edin-A, B and C using the primers described in Table 1 . We have determined optimized thermal cycling conditions for $e$ din-A ( 25 cycles of $30 \mathrm{~s}$ at $94^{\circ} \mathrm{C}, 45 \mathrm{~s}$ annealing at $58^{\circ} \mathrm{C}$ and 1 min elongation at $72^{\circ} \mathrm{C}$ ), edin- $B$ ( 25 cycles of $30 \mathrm{~s}$ at $95^{\circ} \mathrm{C}, 1 \mathrm{~min}$ annealing at $50^{\circ} \mathrm{C}$ and $1 \mathrm{~min}$ elongation at $\left.72^{\circ} \mathrm{C}\right)$ and $e d i n-C(30$ cycles of $30 \mathrm{~s}$ at $94^{\circ} \mathrm{C}, 45 \mathrm{~s}$ annealing at $54^{\circ} \mathrm{C}$ and $1 \mathrm{~min}$ elongation at $72^{\circ} \mathrm{C}$ ). For the detection of other virulence genes, total DNA was isolated from bacterial strains grown three hours at $37^{\circ} \mathrm{C}$ in 
BHI medium. DNA was subsequently extracted with NucleoSpin Tissue (Macherey-Nagel $\mathrm{GmbH}$, Düren, Germany) according to manufacturer's recommendations. Briefly, bacteria were pelleted by centrifugation at $8,000 \times g$ for $5 \mathrm{~min}$, resuspended in $180 \mu 1$ of lysis buffer with $33 \mu \mathrm{l}$ of proteinase $\mathrm{K}(20 \mathrm{mg} / \mathrm{ml})$ (Invitrogen Life Technologies, Carlsbad, CA) and $3 \mu \mathrm{l}$ of

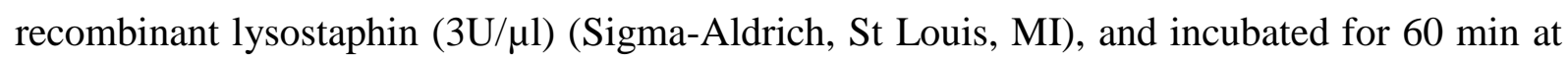
$37^{\circ} \mathrm{C}$. DNA samples were eluted with $100 \mu \mathrm{l}$ alkaline elution buffer (BE buffer, NucleoSpin Tissue, Macherey-Nagel). The presence of 30 genes, among the most prevalent virulenceassociated genes, was evaluated by PCR as described previously: staphylococcal enterotoxins (se) A, B, C, D, E, G, H, I, J, K, and Q, toxic shock syndrome toxin 1 (tst-1), exfoliative toxins A, B and D (etA, etB, etD), PVL (lukS-PV-lukF-PV), LukDE leukocidin (lukE), nine MSCRAMM (bbp, cna, ebpS, clfA, clfB, fib, fnbA, fnbB, eno). The accessory gene regulator (agr) allele group was determined by multiplex PCR.

spa sequencing

spa typing was performed as described previously [23], using the spa typing website (http://www.spaserver.ridom.de/) that is developed by Ridom $\mathrm{GmbH}$ and curated by SeqNet.org (http://www.SeqNet.org/). Primers are indicated in Table 1.

Statistical analysis

The chi-square or Fisher's exact test for categorical variables was used to compare data as appropriate. A $P$ value of less than 0.05 was considered significant. 


\section{Results}

Detection of edin isoforms

S. aureus isolates analyzed in this study were collected at the university hospital of Nice from various infected patients. We designed primers with high sensitivity and specificity in order to detect, by PCR, edin-A, B and C alleles in these strains. This was especially challenging for edin-C, which was poorly detected using a previously described pair of primers designed to amplify all edin isoforms. This is consistent with the fact that the sequence of edin- $C$ has the most substantial sequence variations in regions recognized by these primer sequences $(17 \%$ and $32 \%$ homology with the forward and reverse primers, respectively) (Fig. 1) [19]. As shown in figure 1 , the three pairs of primers designed allowed us to amplify specifically a 455 bp DNA fragment for $e \operatorname{din}-A$ and $B$, and a 320 bp DNA fragment for $e d i n-C$.

We next analyze the 256 clinical strains of $S$. aureus. We found that $14 \%$ (36) of these strains were positive for one of the edin alleles. Among these 36 strains, $90 \%$ were positive for edin-C and $5 \%$ were positive for either edin-A or $B$. To confirm the nature of the edin isoforms, we performed complete sequence analysis of five edin- $C$ encoding genes from randomly selected strains. We also sequenced $e$ din- $A$ and $e$ din- $B$ encoding genes. These results confirm the specificity of the new primers used and unravel that edin- $C$ was more prevalent than other alleles of edin.

Detection of genes encoding virulence factors

The 36 strains positive for edin genes were next analyzed for the distribution of major MSCRAMMs, various staphylococcal enterotoxins, exfoliative toxins, the toxic shock syndrome toxin 1 gene $t s t-1$, as well as leucotoxin family encoding genes. Among the staphylococcal MSCRAMM genes, eno, clfA and clfB were detected in all edin-positive strains (Table 2). bbp, fnbA and ebpS were the less frequently encountered MSCRAMMs among these strains. However these adhesion factors had no preferential distribution among S. aureus isolated from various types of infections. Among the staphylococcal enterotoxins genes the most frequently encountered were seg, sei and sea (Table 2). In addition, $72 \%$ of edin-positive strains (26 strains) presented a combination of these three genes. One edin-C bearing strain, isolated from a urine sample, had only the sea enterotoxin gene. We detected the exfoliative toxin gene $e t D$ exclusively among the edin- $B$ positive strains. Two edin-A 
positive strains carried the staphylococcal exfoliative gene etB. We found that 25 edin- $C$ positive $S$. aureus (78\%) were negative for $e t A$, etB and $e t D$ exfoliative toxin encoding genes. We observed that only $22 \%$ of the edin-C positive $S$. aureus (7 out of 32 ) had at least one of the two isoforms (etA or $e t B$ ) of the exfoliative toxin gene. Five of these seven strains carried both the $e t A$ and $e t B$ genes. Finally, a large number of edin-positive $S$. aureus belonged to the agr group 1 (50\%, 18 strains), and to a lesser extent, to agr groups 2, 3 and 4 (17\%, 6 strains each) (Table 2).

spa-typing

Determination of the spa type of 26 edin-positive strains [23] allowed us to exclude the clonal origin of all edin-C positive $S$. aureus in our survey. Among them, only six strains could be classified as ST45 (two isolates), ST30 (two isolates), ST59 or ST26. The other 20 strains showed a high variability of their spa type (Table 3). For 14 strains, we determined new repeat successions including unidentified 24-bp repeats thus defining new spa types. Among them, strains S7926 and S7262, isolated respectively in deep seated soft tissue and sputum sample from unrelated infected patients, presented the same spa type t6956 (Table 3). Together these data excluded the clonal origin of all edin-C positive $S$. aureus in our survey.

Antibiotic susceptibility profiles

We next investigated whether edin-positive strains were associated with specific antibiotic resistance profiles, such as community-acquired methicillin resistant S. aureus ST80 and fusidic acid-resistant impetigo clones [20,21]. In relation with these studies, we determined the minimum inhibitory concentrations (MICs) of edin-positive strains for the following antibiotics: penicillin G, methicillin, erythromycin, clindamycin and fusidic acid. Fourteen edin-positive strains were susceptible to all tested antimicrobial molecules (41.6\%), except fusidic acid. Indeed, one of 14 isolates tested presented only an increase in fusidic acid MIC, classified as intermediary sensibility. Twenty two isolates were resistant to penicillin G. Among them, 19 were susceptible to all other antimicrobial molecules tested (83\%) and two strains also presented erythromycin resistance (8.7\%). Only one strain, positive for edin-C, showed a methicillin-resistance associated to additional resistance, i.e. erythromycin and clindamycin as well as an increase of MIC to fusidic acid. This strain was negative for etA 
and $e t B$. Finally, edin-positive strains did not show any specific resistance profile to classical antibiotics used to cure infections by $S$. aureus (Table 2 ).

Clinical origin of edin-positive S. aureus

Having shown no link between a high prevalence of edin- $C$ within our $S$. aureus strains and any phenotypic profile or clonal origin, we further analyzed the distribution of edin-positive strains with regard to the infectious sites. We noticed that strains positive for edin- $C$ were recovered at all sites of infection (Table 4). Strikingly, edin-positive $S$. aureus were more significantly associated with deep-seated infections of soft tissues than other types of infections $(25.7 \%$, Fischer exact test, $\mathrm{p}=0.03)$. No significant association was detected between edin-positive or edin-negative strains and other types of infections (blood, urine, superficial soft tissue and sputum culture). 


\section{Discussion}

Our study shows that edin-positive $S$. aureus strains are found in all types of clinical infections included in this study, with a global prevalence of $14 \%$. Moreover, we show that $90 \%$ of edin-positive strains are positive for edin- $C$. This is consistent with a previous study performed with specific primers, which also reported a higher prevalence of $e \operatorname{din}-C$ in clinical strains of S. aureus in Japan [24]. Both studies point for a possible underestimation of the prevalence of edin-C in pathogenic $S$. aureus. This points for the need of using specific primers to detect each edin isoform, especially edin- $C$, given its high prevalence.

Both the analysis of spa type and the distribution of various virulence factors among edin-positive $S$. aureus show their high genetic variability. Our results on the distribution of MSCRAMMs are consistent with previous findings [25]. Classically, edin and exfoliative toxin encoding genes are associated in specific lineages responsible for skin infections [17, $21,24]$. Interestingly, here we show that $e$ din- $C$ is not strictly associated with genes encoding exfoliative toxin of serotypes A/B/D (7 strains edin- $C$-positive and $e$ t-positive, versus 25 strains edin- $C$-positive and $e t$-negative). The high prevalence of edin-C-positive and etnegative strains observed in our study might be explained by the plasticity of the pETB plasmid, which has been previously reported in two different variants [18, 21]. Also, a recent study shows genetic variations in pathogenicity islands encoding EDIN-B [17].

Given that spa analysis constitutes a good tool for epidemiological typing of S. aureus [26], the use of this method allowed us to exclude the clonal origin of edin- $C$ positive strains. The fact that edin- $C$ is plasmid born might explain its presence in strains of various genetic backgrounds.

S. aureus positive for edin are more frequently associated with deep-seated infections of soft tissues. Infection of endothelial cells, and other cell types, by EDIN-producing S. aureus triggers the formation of transcellular tunnels named macroapertures [12, 13]. Moreover, we recently reported that $S$. aureus EDIN toxin promotes formation of infection foci in a mouse model of bacteremia [15]. Together with the present study, this suggests that EDIN might enhance the invasive capacity of $S$. aureus. However, whether or not edin-positive S. aureus is preferentially associated with a specific type of deep seated infection remains to be further determined. 
Acknowledgments We are grateful to Fernand Girard-Pipau and Claire Poyart for providing various strains of $S$. aureus and Pr Jean-Louis Mege for critical reading of the manuscript.

Our laboratory is supported by an institutional funding from the INSERM, a grant from the Agence Nationale de la Recherche (ANR RPV07055ASA) and the Association pour la Recherche sur le Cancer (ARC 4906). 


\section{References}

[1] Lowy FD (1998) Staphylococcus aureus infections. N Engl J Med 339 (8):520-532

[2] Fournier B, Philpott DJ (2005) Recognition of Staphylococcus aureus by the innate immune system. Clin Microbiol Rev 18 (3):521-540

[3] Becker K, Friedrich AW, Lubritz G et al (2003) Prevalence of genes encoding pyrogenic toxin superantigens and exfoliative toxins among strains of Staphylococcus aureus isolated from blood and nasal specimens. J Clin Microbiol 41 (4):1434-1439

[4] Boquet P, Lemichez E (2003) Bacterial virulence factors targeting Rho GTPases: parasitism or symbiosis? Trends Cell Biol 13 (5):238-246

[5] Dinges MM, Orwin PM, Schlievert PM (2000) Exotoxins of Staphylococcus aureus. Clin Microbiol Rev 13 (1):16-34

[6] Wilde C, Aktories K (2001) The Rho-ADP-ribosylating C3 exoenzyme from Clostridium botulinum and related C3-like transferases. Toxicon 39 (11):1647-1660

[7] Wilde C, Vogelsgesang M, Aktories K (2003) Rho-specific Bacillus cereus ADPribosyltransferase C3cer cloning and characterization. Biochemistry 42 (32):9694-9702

[8] Chardin P, Boquet P, Madaule P et al (1989) The mammalian G protein rhoC is ADPribosylated by Clostridium botulinum exoenzyme $\mathrm{C} 3$ and affects actin microfilaments in Vero cells. EMBO J 8 (4):1087-1092

[9] Aktories K, Barbieri JT (2005) Bacterial cytotoxins: targeting eukaryotic switches. Nat Rev Microbiol 3 (5):397-410

[10] Jaffe AB, Hall A (2005) Rho GTPases: biochemistry and biology. Ann Rev Cell Dev Biol 21:247-269

[11] Visvikis O, Maddugoda MP, Lemichez E (2010) Direct modifications of Rho proteins: deconstructing GTPase regulation. Biol Cell 102 (7):377-389

[12] Boyer L, Doye A, Rolando M et al (2006) Induction of transient macroapertures in endothelial cells through RhoA inhibition by Staphylococcus aureus factors. J Cell Biol 173 (5):809-819

[13] Lemichez E, Lecuit M, Nassif X et al (2010) Breaking the wall: targeting of the endothelium by pathogenic bacteria. Nat Rev Microbiol 8 (2):93-104

[14] Rolando M, Munro P, Stefani Cet al (2009) Injection of Staphylococcus aureus EDIN by the Bacillus anthracis protective antigen machinery induces vascular permeability. Infect Immun 77 (9):3596-3601 
[15] Munro P, Benchetrit M, Nahori MA et al (2010) Staphylococcus aureus EDIN toxin promotes formation of indection foci in a mouse model of bacteremia. Infect Immun 78 (8):3404-3411

[16] Inoue S, Sugai M, Murooka Y et al (1991) Molecular cloning and sequencing of the epidermal cell differentiation inhibitor gene from Staphylococcus aureus. Biochem biophys Research Com 174 (2):459-464

[17] Franke GC, Bockenholt A, Sugai M et al (2009) Epidemiology, variable genetic organisation and regulation of the EDIN-B toxin in Staphylococcus aureus from bacteraemic patients. Microbiology 156 (3):860-872.

[18] Yamaguchi T, Hayashi T, Takami H et al (2001) Complete nucleotide sequence of a Staphylococcus aureus exfoliative toxin B plasmid and identification of a novel ADPribosyltransferase, EDIN-C. Infect Immun 69 (12):7760-7771

[19] Czech A, Yamaguchi T, Bader L et al (2001) Prevalence of Rho-inactivating epidermal cell differentiation inhibitor toxins in clinical Staphylococcus aureus isolates. J Infect Dis 184 (6):785-788

[20] Ben Nejma M, Mastouri M, Bel Hadj Jrad B et al (2008) Characterization of ST80 Panton-Valentine leukocidin-positive community-acquired methicillin-resistant Staphylococcus aureus clone in Tunisia. Diagn Microbiol Infectious Dis In press [Epub ahaed of print]

[21] O'Neill AJ, Larsen AR, Skov R, Henriksen AS, Chopra I (2007) Characterization of the epidemic European fusidic acid-resistant impetigo clone of Staphylococcus aureus. J Clin Microbiol 45 (5):1505-1510

[22] Bauer AW, Kirby WM, Sherris JC et al (1966) Antibiotic susceptibility testing by a standardized single disk method. Am J Clin Pathol 45 (4):493-496

[23] Harmsen D, Claus H, Witte W et al (2003) Typing of methicillin-resistant Staphylococcus aureus in a university hospital setting by using novel software for spa repeat determination and database management. J Clin Microbiol 41 (12):5442-5448

[24] Yamaguchi T, Yokota Y, Terajima J et al (2002) Clonal association of Staphylococcus aureus causing bullous impetigo and the emergence of new methicillin-resistant clonal groups in Kansai district in Japan. J Infect Dis 185 (10):1511-1516

[25] Tristan A, Ying L, Bes M et al (2003) Use of multiplex PCR to identify Staphylococcus aureus adhesins involved in human hematogenous infections. J Clin Microbiol 41 (9):4465-4467 
[26] Petersson AC, Olsson-Liljequist B, Miorner H, et al (2010) Evaluating the usefulness of spa typing, in comparison with pulsed-field gel electrophoresis, for epidemiological typing of methicillin-resistant Staphylococcus aureus in a low-prevalence region in Sweden 20002004. Clin Microbiol Infect 16 (5):456-462

[27] Holtfreter S, Bauer K, Thomas D et al (2004) egc-Encoded superantigens from Staphylococcus aureus are neutralized by human sera much less efficiently than are classical staphylococcal enterotoxins or toxic shock syndrome toxin. Infect Immun 72 (7):4061-4071 [28] Johnson WM, Tyler SD, Ewan EP et al (1991) Detection of genes for enterotoxins, exfoliative toxins, and toxic shock syndrome toxin 1 in Staphylococcus aureus by the polymerase chain reaction. J Clin Microbiol 29 (3):426-430

[29] Jarraud S, Mougel C, Thioulouse J et al (2002) Relationships between Staphylococcus aureus genetic background, virulence factors, agr groups (alleles), and human disease. Infect Immun 70 (2):631-641

[30] Lina G, Boutite F, Tristan A et al (2003) Bacterial competition for human nasal cavity colonization: role of Staphylococcal agr alleles. Appl Environmental Microbiol 69 (1):18-23 


\section{Figure legend}

Fig. 1 Characterization of the three edin alleles. A) PCR amplification of edin- $A$, edin- $B$ and edin-C from $S$. aureus strains S25edin- $A(+)[15]$, S7256edin-B(+) and S7475edin-C(+) (this study), respectively, using specific oligonucleotides edinA, edinB, edinC (Table 1) and the previously described edin oligonucleotides referred as edinX [19]. B) Sequence alignments of edin- $A$, edin- $B$ and edin- $C$ showing sequence homologies and localization of each oligonucleotide (underlined: $e \operatorname{din} X$; highlighted: $e \operatorname{din} A, e \operatorname{din} B$ and $e \operatorname{din} C$ ). 
Table 1: Oligonucleotides primers used in this study

\begin{tabular}{|c|c|c|c|}
\hline Gene & Primer sequences & $\begin{array}{l}\text { Size } \\
(\mathrm{kb})\end{array}$ & References \\
\hline \multirow[t]{2}{*}{$\operatorname{edin} \mathrm{A}$} & Sense 5'-GGAGATATTAATAAGCTAGATTC-3' & 455 & This study \\
\hline & Antisense 5'-ATTTTCTTTTTATCATTTGACAATTCT-3' & & \\
\hline \multirow[t]{2}{*}{ edin $\mathrm{B}$} & Sense 5'-GGTGACGTGAACAAATTATCCGA-3' & 455 & This study \\
\hline & Antisense 5'-ATCTTTCTTTTGTTATCAGAAAGTTTA-3' & & \\
\hline \multirow[t]{2}{*}{ edin $\mathrm{C}$} & Sense 5'-CGCCATTAAGGTCTAGTCAAGG-3' & 320 & This study \\
\hline & Antisense 5'-TAGGTCTTCCAGCTAATGCAGC-3' & & \\
\hline \multirow[t]{2}{*}{$b b p$} & Sense 5'-AACTACATCTAGTACTCAACAACAG-3' & 575 & {$[25]$} \\
\hline & Antisense 5'-ATGTGCTTGAATAACACCATCATCT-3' & & \\
\hline \multirow[t]{2}{*}{ cna } & Sense 5'-GTCAAGCAGTTATTAACACCAGAC-3' & 423 & [25] \\
\hline & Antisense 5'-AATCAGTAATTGCACTTTGTCCACTG-3' & & \\
\hline \multirow[t]{2}{*}{$e b p S$} & Sense 5'-CATCCAGAACCAATCGAAGAC-3' & 186 & [25] \\
\hline & Antisense 5'-CTTAACAGTTACATCATCATGTTTATCTTTG-3' & & \\
\hline \multirow[t]{2}{*}{ fnbA } & Sense 5'-GTGAAGTTTTAGAAGGTGGAAAGATTAG -3' & 643 & [25] \\
\hline & Antisense 5'-GCTCTTGTAAGACCATTTTTCTTCAC-3' & & \\
\hline \multirow[t]{2}{*}{$f n b B$} & Sense 5'-GTAACAGCTAATGGTCGAATTGATACT-3' & 524 & [25] \\
\hline & Antisense 5'-CAAGTTCGATAGGAGTACTATGTTC-3' & & \\
\hline \multirow[t]{2}{*}{ fib } & Sense 5'-CTACAACTACAATTGCCGTCAACAG-3' & 404 & [25] \\
\hline & Antisense 5'-GCTCTTGTAAGACCATTTTCTTCAC-3' & & \\
\hline \multirow[t]{2}{*}{ clfA } & Sense 5'-ATTGGCGTGGCTTCAGTGCT-3' & 292 & [25] \\
\hline & Antisense 5'-CGTTTCTTCCGTAGTTGCATTTG-3' & & \\
\hline \multirow[t]{2}{*}{ clfB } & Sense 5'-ACATCAGTAATAGTAGGGGGCAAC-3' & 205 & [25] \\
\hline & Antisense 5'-TTCGCACTGTTTGTGTTTGCAC-3' & & \\
\hline \multirow[t]{2}{*}{ eno } & Sense 5' - ACGTGCAGCAGCTGACT-3' & 302 & [25] \\
\hline & Antisense 5'- CAACAGCATYCTTCAGTACCTTC-3' & & \\
\hline \multirow[t]{2}{*}{ sea } & Sense 5'-GCAGGGAACAGCTTTAGGC-3' & 520 & [27] \\
\hline & Antisense 5'-GTTCTGTAGAAGTATGAAACACG-3' & & \\
\hline \multirow[t]{2}{*}{ seb } & Sense 5'-ATGTAATTTTGATATTCGCAGTG-3' & 683 & [27] \\
\hline & Antisense 5'-TGCAGGCATCATATCATACCA-3' & & \\
\hline sec & Sense 5'-CTTGTATGTATGGAGGAATAACAA-3' & 283 & [27] \\
\hline
\end{tabular}




\begin{tabular}{|c|c|c|c|}
\hline & Antisense 5'-TGCAGGCATCATATCATACCA-3' & & \\
\hline \multirow[t]{2}{*}{ sed } & Sense 5'-GTGGTGAAATAGATAGGACTGC-3' & 384 & [27] \\
\hline & Antisense 5'-ATATGAAGGTGCTCTGTGG-3' & & \\
\hline \multirow[t]{2}{*}{ see } & Sense 5'-TACCAATTAACTTGTGGATAGAC-3' & 170 & [27] \\
\hline & Antisense 5'-CTCTTTGCACCTTACCGC-3' & & \\
\hline \multirow[t]{2}{*}{$\operatorname{seg}$} & Sense 5'-CGTCTCCACCTGTTGAAGG-3' & 327 & [27] \\
\hline & Antisense 5'-CCAAGTGATTGTCTATTGTCG-3' & & \\
\hline \multirow[t]{2}{*}{$\operatorname{seh}$} & Sense 5'-CAACTGCTGATTTAGCTCAG-3' & 360 & [27] \\
\hline & Antisense 5'-GTCGAATGAGTAATCTCTAGG-3' & & \\
\hline \multirow[t]{2}{*}{ sei } & Sense 5'-CAACTCGAATTTTCAACAGGTAC-3' & 465 & {$[27]$} \\
\hline & Antisense 5'- CAGGCAGTCCATCTCCTG-3' & & \\
\hline \multirow[t]{2}{*}{$\operatorname{sej}$} & Sense 5'- CATCAGAACTGTTGTTCCGCTAG-3' & 142 & {$[27]$} \\
\hline & Antisense 5'- CTGAATTTTACCATCAAAGGTAC-3' & & \\
\hline \multirow[t]{2}{*}{ sek } & Sense 5’- ATGGCGGAGTCACAGCTACT-3' & 197 & {$[27]$} \\
\hline & Antisense 5'-TGCCGTTATGTCCATAAATGTT-3' & & \\
\hline \multirow[t]{2}{*}{$\operatorname{seq}$} & Sense 5'-GAACCTGAAAAGCTTCAAGGA-3' & 209 & [27] \\
\hline & Antisense 5'-ATTCGCCAACGTAATTCCAC-3' & & \\
\hline \multirow[t]{2}{*}{ eta } & Sense 5'-CTAGTGCATTTGTTATTCAA-3' & 119 & [28] \\
\hline & Antisense 5'-TGCATTGACACCATAGTACT-3' & & \\
\hline \multirow[t]{2}{*}{$e t b$} & Sense 5'-ACGGCTATATACATTCAATT-3' & 200 & [28] \\
\hline & Antisense 5'-TCCATCGATAATATACCTAA-3' & & \\
\hline \multirow[t]{2}{*}{ etd } & Sense 5'-ATGACTAAAAATATATTAAAAAGTT-3' & 846 & This study \\
\hline & Antisense 5'-CTAATGAGACTGTAATTCAGC-3’ & & \\
\hline \multirow[t]{2}{*}{$l u k P V$} & Sense 5'-ATCATTAGGTAAAATGTCTGGACATGATCCA-3' & 433 & [29] \\
\hline & Antisense 5'-GCATCAASTGTATTGGATAGCAAAAGC-3' & & \\
\hline \multirow[t]{2}{*}{ lukE } & Sense 5'-TGAAAAAGGTTCAAAGTTGATACGAG-3' & 269 & [29] \\
\hline & Antisense 5'-TGTATTCGATAGCAAAAGCAGTGCA-3' & & \\
\hline \multirow[t]{2}{*}{ tst-1 } & Sense 5'-GCTTGCGACAACTGCTACAG-3' & 559 & [27] \\
\hline & Antisense 5'-TGGATCCGTCATTCATTGTTAA-3' & & \\
\hline \multirow[t]{2}{*}{ agrl } & Sense 5'-ATGCACATGG TGCACATGC-3' & 439 & [30] \\
\hline & Antisense 5'-GTCACAAGTACTATAAGCTGCGAT-3' & & \\
\hline \multirow[t]{2}{*}{ agr2 } & Sense 5'-ATGCACATGG TGCACATGC-3' & 572 & {$[30]$} \\
\hline & Antisense 5'-TATTACTAATTGAAAAGTGC CATAGC-3' & & \\
\hline
\end{tabular}




\begin{tabular}{|llrc|}
\hline agr3 & Sense 5'-ATGCACATGG TGCACATGC-3' & 321 & [30] \\
& Antisense 5'-GTAATGTAATAGCTTGTATAATAATACCCAG-3' & & \\
\multirow{2}{*}{ agr4 } & Sense 5'-ATGCACATGG TGCACATGC-3' & 657 & {$[30]$} \\
& Antisense 5'-CGATAATGCCGTAATACCCG-3' & & \\
spa & Sense5' '-TGTAAAACGACGGCCAGTTAAAGACGATCCTTCGGTGAGC-3' & [23] \\
& Antisense5' 'CAGGAAACAGCTATGACCCAGCAGTAGTGCCGTTTGCTT-3'
\end{tabular}


Table 2: Virulence profile and antibiotic susceptibility of clinical edin-positive $S$. aureus strains.

\begin{tabular}{|c|c|c|c|c|c|c|c|c|c|c|c|c|}
\hline & \multicolumn{2}{|c|}{ blood $(n=2)$} & \multicolumn{2}{|c|}{$\begin{array}{c}\text { superficial soft tissue } \\
(n=11)\end{array}$} & \multicolumn{2}{|c|}{ urine sample $(n=4)$} & \multicolumn{2}{|c|}{ sputum sample $(n=10)$} & \multicolumn{2}{|c|}{$\begin{array}{c}\text { deep-seated soft tissue } \\
(n=9)\end{array}$} & \multicolumn{2}{|c|}{ total $(n=36)$} \\
\hline & $\mathrm{n}$ & $\%$ & $\mathrm{n}$ & $\%$ & $n$ & $\%$ & $\mathrm{n}$ & $\%$ & $\mathrm{n}$ & $\%$ & $n$ & $\%$ \\
\hline \multicolumn{13}{|c|}{$\begin{array}{l}\text { Virulence profile } \\
\text { MSCRAMMs }\end{array}$} \\
\hline$b b p$ & 0 & 0 & 3 & 27 & 3 & 75 & 3 & 30 & 6 & 67 & 15 & 42 \\
\hline cna & 1 & 50 & 9 & 82 & 4 & 100 & 10 & 100 & 8 & 89 & 32 & 89 \\
\hline ebps & 0 & 0 & 5 & 45 & 1 & 25 & 6 & 60 & 6 & 67 & 18 & 50 \\
\hline$f n b A$ & 1 & 50 & 7 & 64 & 1 & 25 & 5 & 50 & 5 & 56 & 19 & 53 \\
\hline$f n b B$ & 1 & 50 & 11 & 100 & 4 & 100 & 7 & 70 & 8 & 89 & 31 & 86 \\
\hline fib & 2 & 100 & 10 & 91 & 4 & 100 & 7 & 70 & 9 & 100 & 32 & 89 \\
\hline clfA & 2 & 100 & 11 & 100 & 4 & 100 & 10 & 100 & 9 & 100 & 36 & 100 \\
\hline clfB & 2 & 100 & 11 & 100 & 4 & 100 & 10 & 100 & 9 & 100 & 36 & 100 \\
\hline eno & 2 & 100 & 11 & 100 & 4 & 100 & 10 & 100 & 9 & 100 & 36 & 100 \\
\hline \multicolumn{13}{|l|}{ SEs } \\
\hline sea & 1 & 50 & 10 & 91 & 3 & 75 & 9 & 90 & 9 & 100 & 32 & 89 \\
\hline$s e b$ & 1 & 50 & 7 & 64 & 2 & 50 & 8 & 80 & 6 & 67 & 24 & 67 \\
\hline $\sec$ & 1 & 50 & 7 & 64 & 2 & 50 & 10 & 100 & 6 & 67 & 26 & 72 \\
\hline sed & 0 & 0 & 8 & 73 & 3 & 75 & 6 & 60 & 6 & 67 & 23 & 64 \\
\hline see & 0 & 0 & 0 & 0 & 0 & 0 & 0 & 0 & 0 & 0 & 0 & 0 \\
\hline sek & 1 & 50 & 5 & 45 & 1 & 25 & 7 & 70 & 2 & 22 & 16 & 44 \\
\hline seq & 1 & 50 & 3 & 27 & 1 & 25 & 5 & 50 & 5 & 56 & 15 & 42 \\
\hline seg & 2 & 100 & 10 & 91 & 2 & 50 & 10 & 100 & 9 & 100 & 33 & 92 \\
\hline seh & 0 & 0 & 0 & 0 & 0 & 0 & 0 & 0 & 1 & 11 & 1 & 3 \\
\hline sei & 2 & 100 & 11 & 100 & 2 & 50 & 9 & 90 & 8 & 89 & 32 & 89 \\
\hline sej & 0 & 0 & 1 & 9 & 0 & 0 & 2 & 20 & 1 & 11 & 4 & 11 \\
\hline$t s t-1$ & 2 & 100 & 6 & 55 & 1 & 25 & 7 & 70 & 5 & 56 & 21 & 58 \\
\hline etA & 0 & 0 & 2 & 18 & 0 & 0 & 2 & 20 & 2 & 22 & 6 & 17 \\
\hline etB & $1^{\&}$ & 50 & $4^{\$}$ & 36 & 0 & 0 & 2 & 20 & 1 & 11 & 8 & 22 \\
\hline et $D$ & 0 & 0 & 0 & 0 & $1^{*}$ & 25 & $1^{*}$ & 10 & 0 & 0 & 2 & 6 \\
\hline lukPV & 0 & 0 & 1 & 9 & 1 & 25 & 0 & 0 & 3 & 33 & 5 & 14 \\
\hline lukE & 2 & 100 & 8 & 73 & 3 & 75 & 5 & 50 & 4 & 44 & 24 & 67 \\
\hline \multicolumn{13}{|l|}{ agr group } \\
\hline agr1 & 1 & 50 & 3 & 27 & 3 & 75 & 6 & 60 & 5 & 56 & 18 & 50 \\
\hline agr2 & 1 & 50 & 3 & 27 & 1 & 25 & 1 & 10 & 0 & 0 & 6 & 17 \\
\hline agr3 & 0 & 0 & 3 & 27 & 0 & 0 & 0 & 0 & 3 & 33 & 6 & 17 \\
\hline agr4 & 0 & 0 & 2 & 18 & 0 & 0 & 3 & 30 & 1 & 11 & 6 & 17 \\
\hline \multicolumn{13}{|c|}{ antibiotic resistance profile } \\
\hline penicillin $G$ & 1 & 50 & 5 & 45 & 2 & 50 & 8 & 80 & 6 & 67 & 22 & 61 \\
\hline Methicillin & 0 & 0 & 0 & 0 & 0 & 0 & 0 & 0 & 1 & 11 & 1 & 3 \\
\hline Erythromycin & 0 & 0 & 1 & 9 & 0 & 0 & 1 & 10 & 1 & 11 & 3 & 8 \\
\hline Clindamycin & 0 & 0 & 0 & 0 & 0 & 0 & 0 & 0 & 1 & 11 & 1 & 3 \\
\hline Fusidic acid & 0 & 0 & 0 & 0 & $1 \mathrm{a}$ & 25 & 0 & 0 & $1 \mathrm{a}$ & 11 & 2 & 6 \\
\hline
\end{tabular}


Table 3 : spa-type of 36 edin-positive Staphylococcus aureus isolates.

\begin{tabular}{|c|c|c|c|}
\hline Strains & EDIN type & Spa-type or repeat sequences & MLST \\
\hline \multicolumn{4}{|l|}{ Blood } \\
\hline $\mathrm{S} 25$ & $\mathrm{~A}$ & t6403 & - \\
\hline S7232 & $\mathrm{C}$ & $\mathrm{NT}^{\$}$ & - \\
\hline \multicolumn{4}{|c|}{ Deep-seated soft tissue } \\
\hline S7272 & $\mathrm{C}$ & t6953 & - \\
\hline S7404 & $\mathrm{C}$ & t6649 & - \\
\hline S7408 & $\mathrm{C}$ & t6484 & - \\
\hline S7466 & $\mathrm{C}$ & t012 & ST-30 \\
\hline S7595 & $\mathrm{C}$ & NT & - \\
\hline S7600 & $\mathrm{C}$ & t6677 & - \\
\hline S7926 & $\mathrm{C}$ & t6956 & - \\
\hline S8028* & $\mathrm{C}$ & NT & - \\
\hline S8087 & $\mathrm{C}$ & t6481 & - \\
\hline \multicolumn{4}{|c|}{ Sputum sample } \\
\hline S7225 & $\mathrm{C}$ & $\mathrm{t} 2726$ & - \\
\hline S7259 & $\mathrm{C}$ & $\mathrm{t} 2088$ & - \\
\hline S7262 & $\mathrm{C}$ & t6956 & - \\
\hline S7413 & $\mathrm{C}$ & t6483 & - \\
\hline S7535 & $\mathrm{C}$ & $\mathrm{t} 2647$ & - \\
\hline S7634 & $\mathrm{C}$ & t6954 & - \\
\hline S7649 & $\mathrm{B}$ & NT & - \\
\hline S7920 & $\mathrm{C}$ & NT & - \\
\hline S7965 & $\mathrm{C}$ & NT & - \\
\hline S8100 & $\mathrm{C}$ & t015 & ST-45 \\
\hline \multicolumn{4}{|c|}{ Superficial soft tissue } \\
\hline S7181 & $\mathrm{C}$ & t6650 & - \\
\hline S7183 & $\mathrm{C}$ & NT & - \\
\hline S7436 & $\mathrm{C}$ & t6957 & - \\
\hline S7475 & $\mathrm{C}$ & t6480 & - \\
\hline S7526 & $\mathrm{C}$ & $\mathrm{t} 137$ & - \\
\hline
\end{tabular}




\begin{tabular}{|c|c|c|c|}
\hline S7539 & C & NT & - \\
\hline S7569 & A & t031 & ST-45 \\
\hline S7599 & C & t6482 & - \\
\hline S7932 & C & NT & - \\
\hline S7938 & C & t620 & - \\
\hline S7977 & C & t078 & - \\
\hline Urine sample & t012 & ST-26 \\
\hline S7256 & B & t645 & ST-30 \\
\hline S7322 & C & t216 & ST-59 \\
\hline S7906 & C & C &
\end{tabular}

* MRSA strain

$\$$ Not typable 
Table 4: Presence of edin genes in 256 Staphylococcus aureus strains associated with various clinical syndromes.

\begin{tabular}{lcrccc}
\hline \multirow{2}{*}{ Source of isolates (N) } & \multicolumn{2}{c}{ Number of edin } & \multicolumn{3}{c}{ edin-isoforms } \\
\cline { 3 - 5 } & isolates & (\%) & edinA & edinB & edinC \\
\hline Blood (28) & $2(7.1)$ & 1 & 0 & 1 \\
Urine (41) & $4(9.8)$ & 0 & 1 & 3 \\
Superficial soft tissue (83) & $11(13.3)$ & 1 & 0 & 10 \\
Deep-seated soft tissue (35) & $9(25.7)^{*}$ & 0 & 0 & 9 \\
Sputum (69) & $10(14.5)$ & 0 & 1 & 9 \\
Total (256) & $\mathbf{3 6 ( 1 4 )}$ & $\mathbf{2 ( 5 )}$ & $\mathbf{2 ( 5 )}$ & $\mathbf{3 2}(\mathbf{5 0})$ \\
\hline
\end{tabular}

* $\mathrm{p}<0,05$ (Fisher's exact test) 


\section{Figure1}

$3 \quad \begin{aligned} & \mathrm{EDIN}-\mathrm{C} \\ & \mathrm{EDIN}-\mathrm{B}\end{aligned}$

\section{A Specific primers}

\section{Strains}

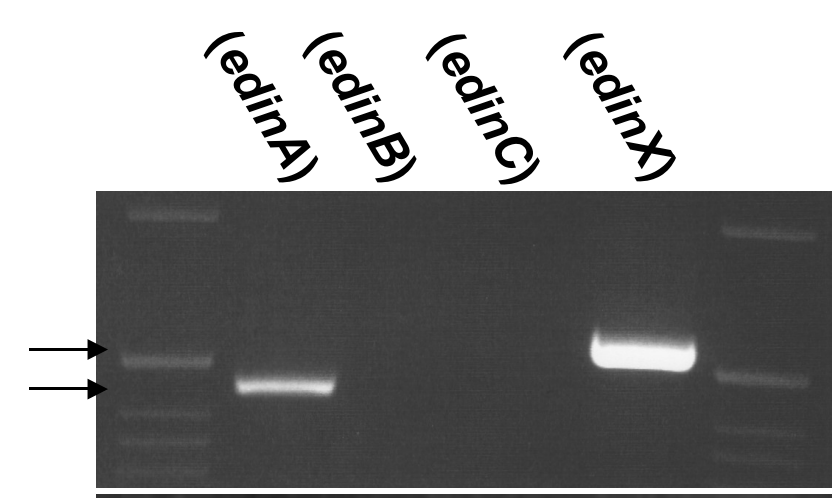

$\$ 7256$ edin-B(+)

$S 7475$ edin-C(+)
ATGAAAAACA.AATTACTTTTTA.AATTTTTTTGAGTTTA TC TTTAGCATT ATGAAAAGAAAATTA TTTT TTAAAATTA TTTTTG TTTTA TC TTTAGTA TT ATGAAAGATA-- CAATTGTAAAATTTCTA TCAGCATTCCTTGTGATTTC AAGCGTTTATTCAATTAATGA----TAAAA TCATAGAAGTATC TAATACT AAGCATTCATTCAATTAATGA----CAGAACTACAGAGTTATCAAACATT ARGTATTAGTTTGATAGATACATC TTTTAGCTCTAAATATAATAAA-ATC * * + t+ *t * +

TC TTTAGCAGCTGATGTTAAAAATTCACTGATTTAGATGAGGCAACTAA GC TTTA GCAGATGATGTTAAAAATTTTACC GA TTTAACTGAAGCAACTAA TCAA TAGCTGCCGAGACTAAAAATTTTACAGACTTAGTTGAAGCTACTAA

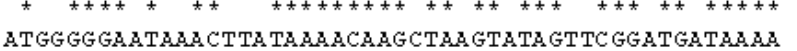
ATGGGGGAATA_ACTTA TAAAACAAGCTAAGTATAGTTCGGATGATAAAA CTGGGGTAATAAGCTTA TAAAACAAGCTAA TTACA GTTCAAAA GACAAAG T

TAGCTC TA TA CGAATATACAAARGATAGTTCTABGATAAATGGTCCATTA AA GC TA TTTA TAA TTATACAAAATATAGCTCGCCTATAAATACGCCATTA TGGC TA TTTA TAA TTATACAAAAAATAG TTCACCCATAAATAC TCCTC TA

GACTCGCAGGTGGAGATATTARTAMGCTAGATTCAMCAACTCAMGACA AGGTCTAGTCARGGTGATA TAAGTAA TTTTTC TGCAGATTTACAAGAAA

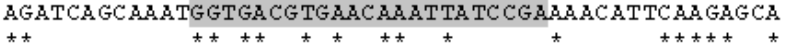
AGTAAGAAGA TTAGATTCATCTA TTTCTAAATCTACTACTCCTGAATCTG AA TACTTC GATTA GA TA GACTCATAA GCAAATCAAGTACTAGTGATTC TG GGTTAGACAA TTA GACTCAAC GATATCTAAATCTGTAACACCA GATTCAG

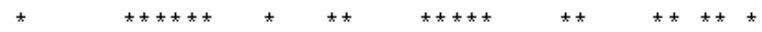
TA TACGTTTA TAGACTTTTAAATTTAGATTATTTGACAAGTATCGTTGGA TA TA TG TTTA TAGATTGCTAAATC TGGACTATTTA TCCAGTGTTAAAGGT TC TA TG TA TA TAGATTA TTAAATTTAGACTAC TTA TCAAGTATAACTGGC

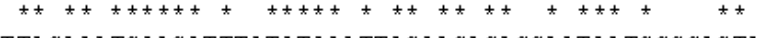
TTTACAAA TGAAGATTTATATAAATTACAACAGACCAATAATGGCCAGTA TTTTCTTC TGAAGATTTGGAA TTA TTATACAAAACAGAAAA TGGTAAGTA TTTACGCGAGAAGATTTACATATGCTACAACAAAC TAACAA TGGTCAATA

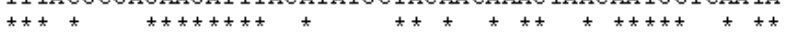
TGATGAAAATCTAGTTAGAARGCTTAATAACGTTA TGAATA GCAGARTAT TA.ATGAAGAB TTAGTTABAAABCTTA.ATAA TA TTA TGAA TA GTAA.AATTT TGATGAAGCGCTTGTGTCAAAACTAAATAA TC TTA TGAA TA GTAGAATTT

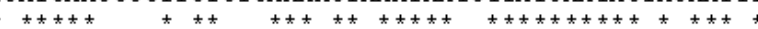
ATAGAGAAGACGGATACTCTAGTACACAATTAGTTAGTGGAGCAGCTGTA ATAC TGAGTACGGTTATTC TA GCACTCAATTA GTTAAAGGA GCTGCATTA

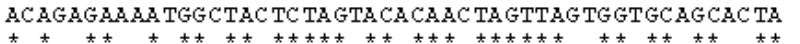
GGTGGTAGACCTA TTGAATTAAGGTTAGAATTACCAAAAGGGACTAAAGC GC TGGAAGACCTA TTGAATTGAAA TTAC AA TTACCAAAAGG TACTABAGC GCAGGTAGGCCAA TTGAATTAAAA TTAGAA TTACC TAAAGGTACTAAAGC

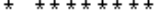
TGCGTA TCTTAATTC TAAAGA TTTAACTGCTTACTATGGTCAACAAGAA AGCATATATTGATTC TAAAGAGTTAACAGCATACCCAGGTCAACAAGAAG

TTTTATTACC TAGAGGCACAGAATACGC TGTTGGAAGTGTAGAATTGTCA TA TTGTTGCCTAGAGGAACAGACTACAC TA TAAATACA GTCAAAC TTTCA TTCTTTTGCC TAGAGGGACAGAATATGC TGTAGGCAGTGTTAAACTTTCT

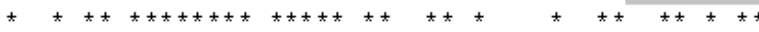
AATGATAAAAAGAABATCATAATAACAGCTATTGTTTTTAAAAARTAG GATGATCATAAAAGAATTTAATCGAAGGTATCGTTTTCAAAAAGTAM GA TAACAAAA GAAAGATAA TTATAACTGCTGTAGTTTTTAAAAAATAA 


\section{Figure1}

$3 \quad \begin{aligned} & \mathrm{EDIN}-\mathrm{C} \\ & \mathrm{EDIN}-\mathrm{B}\end{aligned}$

\section{A Specific primers}

\section{Strains}

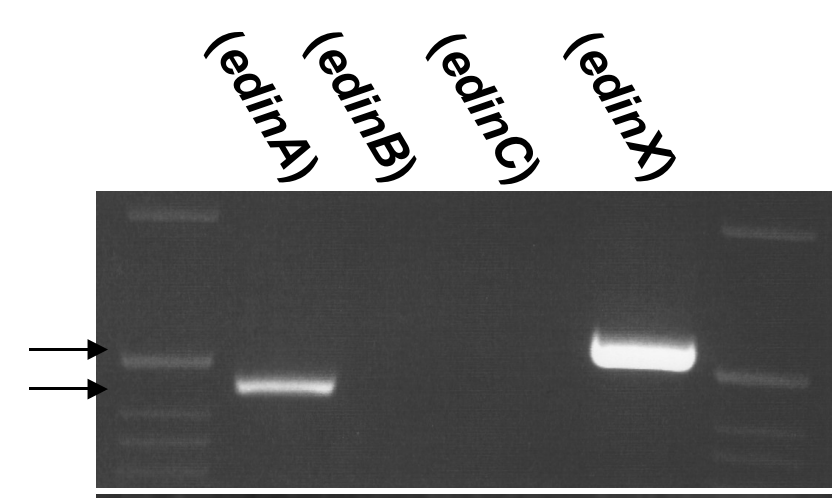

$\$ 7256$ edin-B(+)

$S 7475$ edin-C(+)
ATGAAAAACA.AATTACTTTTTA.AATTTTTTTGAGTTTA TC TTTAGCATT ATGAAAAGAAAATTA TTTT TTAAAATTA TTTTTG TTTTA TC TTTAGTA TT ATGAAAGATA-- CAATTGTAAAATTTCTA TCAGCATTCCTTGTGATTTC AAGCGTTTATTCAATTAATGA----TAAAA TCATAGAAGTATC TAATACT AAGCATTCATTCAATTAATGA----CAGAACTACAGAGTTATCAAACATT ARGTATTAGTTTGATAGATACATC TTTTAGCTCTAAATATAATAAA-ATC * * + t+ *t * +

TC TTTAGCAGCTGATGTTAAAAATTCACTGATTTAGATGAGGCAACTAA GC TTTA GCAGATGATGTTAAAAATTTTACC GA TTTAACTGAAGCAACTAA TCAA TAGCTGCCGAGACTAAAAATTTTACAGACTTAGTTGAAGCTACTAA

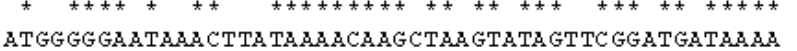
ATGGGGGAATA_ACTTA TAAAACAAGCTAAGTATAGTTCGGATGATAAAA CTGGGGTAATAAGCTTA TAAAACAAGCTAA TTACA GTTCAAAA GACAAAG T

TAGCTC TA TA CGAATATACAAARGATAGTTCTABGATAAATGGTCCATTA AA GC TA TTTA TAA TTATACAAAATATAGCTCGCCTATAAATACGCCATTA TGGC TA TTTA TAA TTATACAAAAAATAG TTCACCCATAAATAC TCCTC TA

GACTCGCAGGTGGAGATATTARTAMGCTAGATTCAMCAACTCAMGACA AGGTCTAGTCARGGTGATA TAAGTAA TTTTTC TGCAGATTTACAAGAAA

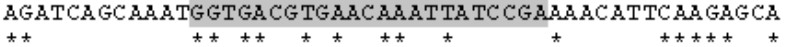
AGTAAGAAGA TTAGATTCATCTA TTTCTAAATCTACTACTCCTGAATCTG AA TACTTC GATTA GA TA GACTCATAA GCAAATCAAGTACTAGTGATTC TG GGTTAGACAA TTA GACTCAAC GATATCTAAATCTGTAACACCA GATTCAG

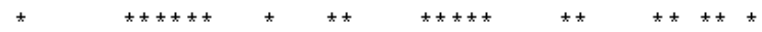
TA TACGTTTA TAGACTTTTAAATTTAGATTATTTGACAAGTATCGTTGGA TA TA TG TTTA TAGATTGCTAAATC TGGACTATTTA TCCAGTGTTAAAGGT TC TA TG TA TA TAGATTA TTAAATTTAGACTAC TTA TCAAGTATAACTGGC

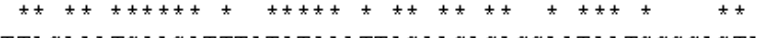
TTTACAAA TGAAGATTTATATAAATTACAACAGACCAATAATGGCCAGTA TTTTCTTC TGAAGATTTGGAA TTA TTATACAAAACAGAAAA TGGTAAGTA TTTACGCGAGAAGATTTACATATGCTACAACAAAC TAACAA TGGTCAATA

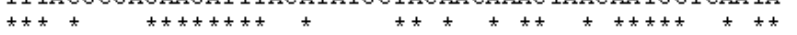
TGATGAAAATCTAGTTAGAARGCTTAATAACGTTA TGAATA GCAGARTAT TA.ATGAAGAB TTAGTTABAAABCTTA.ATAA TA TTA TGAA TA GTAA.AATTT TGATGAAGCGCTTGTGTCAAAACTAAATAA TC TTA TGAA TA GTAGAATTT

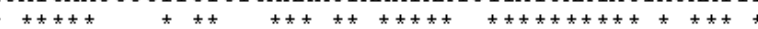
ATAGAGAAGACGGATACTCTAGTACACAATTAGTTAGTGGAGCAGCTGTA ATAC TGAGTACGGTTATTC TA GCACTCAATTA GTTAAAGGA GCTGCATTA

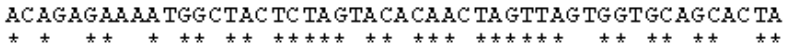
GGTGGTAGACCTA TTGAATTAAGGTTAGAATTACCAAAAGGGACTAAAGC GC TGGAAGACCTA TTGAATTGAAA TTAC AA TTACCAAAAGG TACTABAGC GCAGGTAGGCCAA TTGAATTAAAA TTAGAA TTACC TAAAGGTACTAAAGC

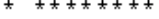
TGCGTA TCTTAATTC TAAAGA TTTAACTGCTTACTATGGTCAACAAGAA AGCATATATTGATTC TAAAGAGTTAACAGCATACCCAGGTCAACAAGAAG

TTTTATTACC TAGAGGCACAGAATACGC TGTTGGAAGTGTAGAATTGTCA TA TTGTTGCCTAGAGGAACAGACTACAC TA TAAATACA GTCAAAC TTTCA TTCTTTTGCC TAGAGGGACAGAATATGC TGTAGGCAGTGTTAAACTTTCT

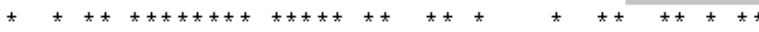
AATGATAAAAAGAABATCATAATAACAGCTATTGTTTTTAAAAARTAG GATGATCATAAAAGAATTTAATCGAAGGTATCGTTTTCAAAAAGTAM GA TAACAAAA GAAAGATAA TTATAACTGCTGTAGTTTTTAAAAAATAA 